\title{
The Role of Thermodynamics in Model Reduction when Using Invariant Grids
}

\author{
Eliodoro Chiavazzo ${ }^{1}$, Iliya V. Karlin ${ }^{1,2, *}$ and Alexander N. Gorban ${ }^{3}$ \\ ${ }^{1}$ Aerothermochemistry and Combustion Systems Lab, ETH Zurich, \\ 8092 Zurich, Switzerland. \\ 2 School of Engineering Sciences, University of Southampton, SO17 1BJ \\ Southampton, United Kingdom. \\ ${ }^{3}$ Department of Mathematics, University of Leicester, LE1 7RH Leicester, \\ United Kingdom.
}

Received 3 July 2009; Accepted (in revised version) 21 January 2010

Available online 17 May 2010

\begin{abstract}
In the present work, we develop in detail the process leading to reduction of models in chemical kinetics when using the Method of Invariant Grids (MIG). To this end, reduced models (invariant grids) are obtained by refining initial approximations of slow invariant manifolds, and used for integrating smaller and less stiff systems of equations capable to recover the detailed description with high accuracy. Moreover, we clarify the role played by thermodynamics in model reduction, and carry out a comparison between detailed and reduced solutions for a model hydrogen oxidation reaction.
\end{abstract}

AMS subject classifications: 52B10, 65D18, 68U05, 68U07

Key words: Chemical kinetics, model reduction, invariant manifold, entropy.

\section{Introduction and motivation}

The numerical solution of the full set of governing equations, as dictated by modeling of reactive flows with detailed chemical kinetics, remains a challenging task. The reason is, on one side, a large number of kinetic equations needs to be solved in order to keep track of each chemical species. On the other side, a detailed reaction mechanism typically contains many different chemical processes occurring on timescales that range over several orders of magnitude, from seconds down to nanoseconds. It is this feature that gives rise to the stiffness of the governing equations for the chemical reactions. Moreover, the fluid mechanics of chemically reactive flows usually occurs at a narrower range on the

*Corresponding author. Email addresses: eliodoro.chiavazzo@gmail.com (E. Chiavazzo), karlin@lav . mavt.ethz.ch (I. V. Karlin), ag153@leicester.ac.uk (A. N. Gorban) 
order of milliseconds to microsecond. The stiffness drastically affects the implementation of numerical solvers, where reducing the time step becomes necessary in order to both avoid numerical instabilities (e.g., in the case of explicit schemes) and to keep a satisfactory accuracy. As a result, the smallest time scales need to be resolved even when one is interested only in the slow dynamics. In addition, the larger the number of elementary reactions involved in the detailed mechanism, the more significant becomes the computational effort due to the evaluation of reaction rates. These issues make computations of even simple reacting mixtures time consuming.

On the other hand, some reduction is often possible by simply setting up a criterion for eliminating unimportant reactions (or species) from the detailed reaction mechanism (see, e.g., the sensitivity analysis [1,2], the comparative analysis of entropy production [3,4], and the reaction path analysis [5]). However, in the present study we exploit a more sophisticated concept of time-scale separation in order to construct a reduced description of the detailed kinetic model. In fact, as mentioned above, there are many chemical processes that are much faster than the fluid dynamic phenomena, so if we are only interested in computing the system behavior on the scale of the fluid mechanics, some chemical processes will be already self-equilibrated. The timescale-based reduction techniques are all based on decoupling the fast equilibrating chemical processes from the slower dynamics, and are implemented by seeking a low dimensional manifold of slow motions in the solution space of the detailed system.

In this work, we focus on closed isothermal chemical reactors with a unique steady state (equilibrium point). However, notice that applications of reduced models attained by the suggested methodology to open reactive systems, under non-isothermal conditions, have been shown recently $[20,21]$.

The idea that a low dimensional manifold provides a reduced description of a complex system stems from the representation of its numerical solutions in the phase-space (concentration space). The dynamics of such a complex reactive system is often characterized by a short initial transient during which the solution trajectories approach lowdimensional manifolds in the concentration space, known as the slow invariant manifolds (SIM). The remaining dynamics lasts much longer and evolves along the SIM towards the steady state. Thus, it turns out that constructing the SIM enables to establish a simplified description of a complex system by extracting only the slow dynamics and neglecting the fast. As a result, the detailed large set of equations can be reduced to a much smaller system without a significant loss of accuracy.

Therefore, much effort has been devoted to setting up automated model reduction procedures based (explicitly or implicitly) on the notion of SIM: The method of invariant grids (MIG) [6,7], the computational singular perturbation (CSP) method [29-31], the intrinsic low dimensional manifold (ILDM) [27, 28], the invariant constrained equilibrium edge preimage curve method (ICE-PIC) [32], the equation-free approaches [33], the method of minimal entropy production trajectories (MEPT) [35] and minimum curvature [36], the constrained runs algorithm in [34] and the finite-time Lyapunov analysis in [37] are some representative examples. 
This work is organized in sections as follows. In Section 2, the notions of invariant manifold and slow invariant manifold are discussed. In Section 3, we briefly outline the kinetic equations for reactive systems. Dissipative stiff systems and the notion of thermodynamic projector are discussed in Section 4. In Section 5, we review the method of invariant grids and the quasi-equilibrium grid algorithm. The above ideas are discussed with the help of two illustrative example in Section 6. In Section 7, we present the parameterization of invariant grids and numerical integration of reduced dynamical systems while, in Section 8, the issue of reducing an arbitrary off-manifold initial condition is addressed. Finally, conclusions are drawn in Section 9.

\section{Slow invariant manifold (SIM)}

In this section, we discuss the notions of (positively) invariant manifold, slow invariant manifold, invariant grid, and slow invariant grid, for a general system of autonomous ordinary differential equations in a domain $\mathcal{D}$ in $\boldsymbol{R}^{n}$,

$$
\dot{c}=J(c) .
$$

\subsection{Invariant manifold and invariance equation}

A submanifold $\Omega \subset \mathcal{D}$ is a positively invariant manifold for the system (2.1) if, for any solution $c(t)$, inclusion $c\left(t_{0}\right) \in \mathbf{\Omega}$, implies that

$$
c(t) \in \Omega, \text { for } t>t_{0} .
$$

Such a set $\Omega$ is often called an invariant manifold.

For each point $c \in \Omega$, the tangent space $T_{c} \Omega$ is defined. If $\Omega$ is positively invariant with respect to system (2.1), then vector $J(c)$ belongs to this tangent space. This gives us a necessary differential condition of invariance

$$
J(c) \in T_{c} \Omega .
$$

In the sequel, because $\Omega$ is unambiguously defined, to save notation we write $T_{c}$ instead of the full notation $T_{c} \Omega$. In order to transform the inclusion condition (2.2) into an equation, we need to execute the following steps:

1. to take a complement to $T_{\mathbf{c}}$ in $\boldsymbol{R}^{n}, \boldsymbol{R}^{n}=T_{\mathbf{c}} \oplus E_{\mathbf{c}}$,

2. to split $\boldsymbol{J}(\boldsymbol{c})$ into two components: $\boldsymbol{J}(\boldsymbol{c})=\boldsymbol{J}_{\|}(\boldsymbol{c})+\boldsymbol{J}_{\perp}(\boldsymbol{c}), \boldsymbol{J}_{\|}(\boldsymbol{c}) \in T_{\mathbf{c}}, \boldsymbol{J}_{\perp}(\boldsymbol{c}) \in E_{\mathbf{c}}$,

3. to write down an equation $J_{\perp}(c)=0$.

These operations are conveniently described by means of projector operators. Let for any subspace $T_{c}$ a projector $\boldsymbol{P}$ on $T_{c}$ be defined with

$$
\text { image } \operatorname{im} \boldsymbol{P}=T_{c}, \quad \text { kernel } \operatorname{ker} \boldsymbol{P}=E_{\boldsymbol{c}} \text {. }
$$


Then the necessary differential condition of invariance takes the following form

$$
(1-\boldsymbol{P}) \boldsymbol{J}=0 .
$$

The left-hand side of this equation is important for many constructions and has its own name, the defect of invariance:

$$
\Delta=J_{\perp}=(1-P) J .
$$

The unknown in this invariance equation (2.3) is the manifold $\Omega$. This manifold has to be represented in a parametric form, as an immersion $F: \mathcal{W} \rightarrow \mathcal{D}$ of a domain $\mathcal{W}$ in the parameter space into the domain $\mathcal{D} ; \Omega$ is the image of this immersion: $\Omega=F(\mathcal{W})$. The tangent space at the point $F(y)$ is the image of the differential of $F$ at the point $y$. Hence, Eq. (2.3) is a differential equation for $F$. The theory of analytic solutions of this equation with analytic vector field $J$ near an equilibrium was developed by Lyapunov [39] (the Lyapunov auxiliary theorem). Applications of this theorem to model reduction were developed recently [9].

Projector $\boldsymbol{P}$ depends on the point $\boldsymbol{c}$ and the space $T_{\boldsymbol{c}}$. Invariance equations for different choices of this projector field $\boldsymbol{P}$ are equivalent, the only requirement is $\operatorname{im} \boldsymbol{P}=T_{\boldsymbol{c}}$. But the convergence properties of computational methods significantly depend on the projector choice. The definition of slowness can also be sensitive to this choice.

At a first glance, there exists a natural method for projector field $\boldsymbol{P}$ construction: If for any $c$ a positive definite inner product $\langle x, y\rangle_{c}$ (a Riemannian structure) is defined, then we can choose $\boldsymbol{P}$ as $\langle,\rangle_{c}$-orthogonal projector, and

$$
J(c)=J_{\|}(c)+J_{\perp}(c),
$$

is $\langle,\rangle_{c}$-orthogonal splitting. A more careful analysis shows that this idea is "almost true", and after some modifications leads to the thermodynamic projector $[23,25]$ (see Section 4.2 below). The relevant Riemannian structure is generated by the second differential of the entropy.

In a majority of applications, we are looking not for an approximation to an invariant manifold that definitely exists, but rather for an approximate invariant manifold with sufficiently small defect of invariance $\Delta(\|\Delta\| \ll\|J\|$, for example).

\subsection{Slow manifold}

Reduction of description for dissipative kinetics assumes (explicitly or implicitly) the following picture (Fig. 1): There exists a manifold of slow motions $\Omega_{\text {slow }}$ in the phase-space. From the initial conditions the system goes quickly in a small neighborhood of the manifold, and after that moves slowly along it. The manifold of slow motions (slow manifold, for short) must be positively invariant: If a solution starts on the manifold at $t_{0}$, then it stays on the manifold at any future time $t>t_{0}$. In some neighborhood of the slow manifold the directions of fast motion can be defined. Of course, we always deal not with the invariant slow manifold, but with some approximate slow manifold $\Omega$. 


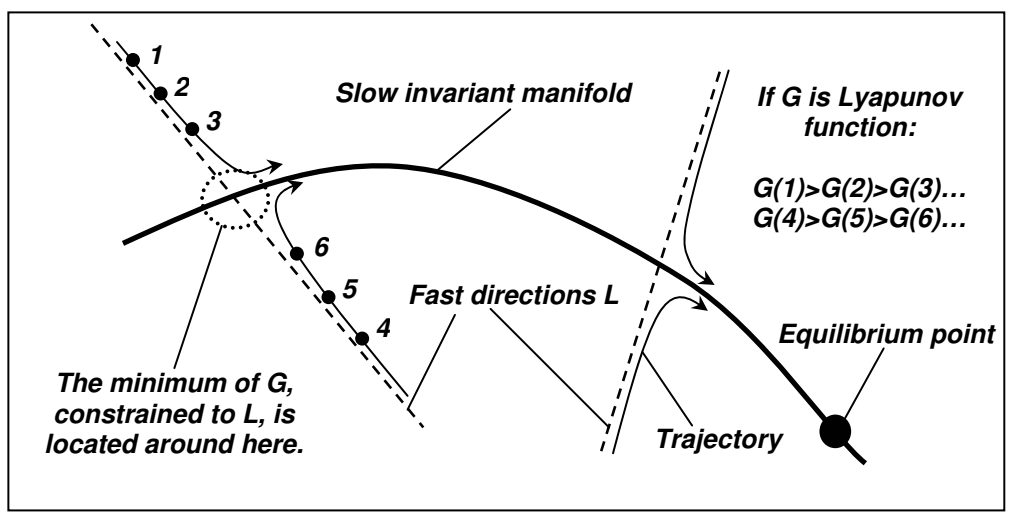

Figure 1: Slow-fast motion decomposition and the existence of a thermodynamic Lyapunov function G.

Thermodynamics is useful for model reduction in dissipative systems. The governing idea of these applications is [23]: During the fast motion the entropy should increase, hence, the point of entropy maximum on the plane of rapid motion is not far from the slow manifold, in the area where fast and slow motion have comparable velocities (Fig. 1, inside dashed circles). This implies that differential of the entropy at points near the slow manifold almost annuls the planes of fast motions (i.e., entropy gradient is almost orthogonal to these planes). For sufficiently strong fast-slow time separation the fast invariant subspace of a Jacobian near the slow manifold approximates the plane of fast motions, hence, this invariant subspace is also nearly orthogonal to the entropy gradient. See also the illustrative examples in Section 4.

All the definitions of slow manifold for a given system are based on the comparison of motion to the manifold with motion along the manifold. There should be relatively fast contraction in selected transversal directions (in directions of projector kernel) and relatively slow change of vector field tangent component along manifold. In this paper, we do not review all these approaches (the spectral gap condition, the cone condition, various stability conditions), the details and further references are in [10-14].

For our approach, the slow invariant manifold is the stable fixed point of one of the following processes:

1. Relaxation due to a film extension of dynamics [17], that is defined by the equation for immersed manifold motion with velocity $J_{\perp}(c)$,

$$
\frac{\mathrm{d} F(y)}{\mathrm{d} t}=(1-P) J
$$

2. Iterations of the Newton method with incomplete linearization for invariance equation (2.3), that is the Newton process without linearization of $\boldsymbol{P}$ : We take $\boldsymbol{J}$ in the first approximation, while for $\boldsymbol{P}$ use the zeroth approximation (for details see Section 5.2). 
If the Newton method with incomplete linearization converges, then it leads to slow manifold in the usual sense while the standard Newton method does not. (This is convenient because the standard Newton method is also more complicated.) For sufficiently strong fast-slow time separation, most of the numerous definitions of slow invariant manifold give the same result (exactly the same, or up to higher order terms, it depends on the required regularity of manifolds) [17].

If we have found an approximate slow invariant manifold $\Omega$, then the corresponding slow reduced system is the system on the manifold $\Omega$ defined by the projected vector field

$$
\dot{\boldsymbol{c}}=\boldsymbol{P J}(\boldsymbol{c}),
$$

where $\boldsymbol{c} \in \boldsymbol{\Omega}$ and projector $\boldsymbol{P}: \boldsymbol{R}^{n} \rightarrow T_{c} \boldsymbol{\Omega}$ depends on the point $\boldsymbol{c}$ and on the tangent space $T_{c} \Omega$, both. Because $F$ is an immersion, the differential of $F(y), D F(y)$, is reversible on its image, $T_{F(y)} \Omega$. Hence, reduced system (2.5) defines dynamics in the parameter space

$$
\dot{y}=(D F(y))^{-1}(\boldsymbol{P J}(F(y))) .
$$

\section{Dissipative reaction kinetics}

In a closed system with $n$ chemical species $A_{1}, \cdots, A_{n}$, participating in a complex reaction, a generic reversible reaction step can be written in the form of a stoichiometric equation

$$
\alpha_{s 1} A_{1}+\cdots+\alpha_{s n} A_{n} \rightleftharpoons \beta_{s 1} A_{1}+\cdots+\beta_{s n} A_{n}
$$

where $s$ is the reaction index, $s=1, \cdots, r$ ( $r$ steps in total), and the integers $\alpha_{s i}$ and $\beta_{s i}$ are stoichiometric coefficients of the step s. For each step, we can introduce $n$-component vectors $\boldsymbol{\alpha}_{s}$ and $\boldsymbol{\beta}_{s^{\prime}}$, with components $\alpha_{s i}$ and $\beta_{s i}$, and the stoichiometric vector $\gamma_{s}=\beta_{s}$ $\boldsymbol{\alpha}_{s}$. For every $A_{i}$ the extensive variable $N_{i}$ describes the number of moles of species $i$. If $V$ is the reactor volume, then the molar concentration of $A_{i}$ is given by $c_{i}=N_{i} / V$. Species concentrations evolve in time, according to the stoichiometric mechanism (3.1), as follows:

$$
\dot{N}=V \boldsymbol{J}(\boldsymbol{c}), \quad \boldsymbol{J}(\boldsymbol{c})=\sum_{s=1}^{r} \gamma_{s} W_{s}(\boldsymbol{c}),
$$

where the dot denotes the time derivative and $W_{s}(\boldsymbol{c})$ is the reaction rate function of the step s. In particular, the polynomial form of the reaction rate function is provided by the mass action law

$$
W_{s}(\boldsymbol{c})=W_{s}^{+}(\boldsymbol{c})-W_{s}^{-}(\boldsymbol{c})=k_{s}^{+}(T) \prod_{i=1}^{n} c_{i}^{\alpha_{i}}-k_{s}^{-}(T) \prod_{i=1}^{n} c_{i}^{\beta_{i}},
$$

where $k_{s}^{+}(T)$ and $k_{s}^{-}(T)$ are the constants of the direct and of the inverse reactions rates of the step $s$, respectively. The most popular form of their dependence is given by the Arrhenius equation

$$
k_{s}^{ \pm}(T)=a_{s}^{ \pm} T^{b_{s}^{ \pm}} \exp \left(S_{s}^{ \pm} / k_{B}\right) \exp \left(-H_{s}^{ \pm} / k_{B} T\right) .
$$


In the latter equation, $a_{s}^{ \pm}, b_{s}^{ \pm}$are constants and $H_{s}^{ \pm}, S_{s}^{ \pm}$activation enthalpies and entropies, respectively. The rate constants are not independent, and the principle of detail balance establishes the relation between those quantities

$$
W_{s}^{+}\left(c^{e q}\right)=W_{s}^{-}\left(c^{e q}\right), \quad \forall s=1, \cdots, r,
$$

where the non-negative vector $c^{e q}$ is the steady state (equilibrium point) of the system (3.2). In general, in order to solve the kinetic equations (3.2), additional conditions are needed. For instance, in the case of isolated reactors, the additional equations

$$
\bar{u}, V=\text { const, }
$$

where $\bar{u}$ is the internal energy, must be taken into account, whereas for an isochoric isothermal system we impose $V, T=$ const, and so forth. However, in the latter case, Eq. (3.2) takes a closed form

$$
\dot{c}=\sum_{s=1}^{r} \gamma_{s} W_{s}(\boldsymbol{c})=\boldsymbol{J}(\boldsymbol{c}) .
$$

Finally, also other linear constraints, related to the conservation of atoms, must be considered. In general, such conservation laws can be written as follows:

$$
\text { Dc }=\text { const }
$$

where $l$ fixed and linearly independent vectors $\boldsymbol{d}_{i}$ are the rows of the $l \times n$ matrix $D$, and const is a constant vector. More details about chemical kinetics can be found, e.g., in the classical work of Williams [8].

\section{Dissipative systems with disparate time scales}

In the present work, we deal with the kinetic equations (3.6) describing time evolution of isochoric isothermal closed reactors towards the unique steady state, $c^{\text {eq }}$, in the interior of the phase-space. Moreover, let us assume that the dynamics induced by the vector field $J$ is characterized by different time scales. In other words, let the Jacobian matrix of (3.6) posses non-positive eigenvalues with different orders of magnitude. In this case, relaxation of solution trajectories, in the phase space, presents a remarkable behaviour: After a short initial transient, they reach the same low dimensional manifold $\Omega$. In the remaining evolution, which lasts much longer, trajectories do not leave $\Omega$ any more, while proceeding towards the steady state (see Fig. 1). In general, the slow motions of (3.6) are relevant for its macroscopic description. In this case, since those motions are "anchored" to the SIM, only $m$ equations of (3.6) are independent ( $m$ is the SIM dimension): The minimal subset of independent equations is the reduced system, while the rest of variables $(n-m)$ respect some relations dictated by the SIM equation. The final aim in model reduction is to obtain the reduced system (smaller and less stiff than the detailed one) to solve instead of (3.6). According to the MIG method, the following steps need to be executed [7]: 
1. Initial grid construction;

2. Grid refinements;

3. Invariant grid parameterization;

4. Reduction of both the system (3.6) and its initial condition.

\subsection{Thermodynamic Lyapunov function}

Our scope is to illustrate the full model reduction process, and to this end, we need to discuss some properties of dynamical systems characterized by disparate time scales and supported by Lyapunov functions.

Let a Lyapunov function $G$ with respect to (3.6) be defined, such that the following dissipation inequality holds

$$
\dot{G}(c)=(\nabla G, J) \leq 0 .
$$

Based on the ideas in Section 2.2, the introduction of $G$ is convenient for model reduction, and in the next sections we intend to show that, when such a function $G$ is defined and the invariant grid constructed, the dynamics induced by the vector field $J$ can be decomposed in terms of slow and fast motions on the basis of thermodynamics.

There are four classical conditions in thermodynamics where the Lyapunov function for the kinetic equation is well known:

\footnotetext{
1. Isolated systems (fixed volume $V$ and internal energy $\bar{u}$ );

2. Isobaric isenthalpic systems (fixed pressure $p$ and enthalpy $\bar{h}$ );

3. Isobaric isothermal systems (fixed pressure $p$ and temperature $T$ );

4. Isochoric isothermal systems (fixed volume $V$ and temperature $T$ ).
}

When dealing with both isolated and isobaric isenthalpic systems, $G$ is related to the mixture averaged entropy $\bar{s}$ which, for ideal gas mixtures, takes the following explicit form:

$$
G_{V, \bar{u}}=G_{p, \bar{h}}=-\bar{s}=-\frac{1}{\bar{W}} \sum_{i=1}^{n}\left[s_{i}(T)-\mathcal{R} \ln \left(X_{i}\right)-\mathcal{R} \ln \left(\frac{p}{p_{r e f}}\right)\right] X_{i}
$$

where $\bar{W}, \mathcal{R}, X_{i}, p$ and $p_{\text {ref }}$ denote the mean molecular weight, the universal gas constant, the mole fraction of species $i$, the total pressure and a reference pressure, respectively. The specific entropy $s_{i}$, according to [26], is assumed to have the following dependence on the temperature $T$

$$
s_{i}(T)=\mathcal{R}\left(a_{i 1} \ln T+a_{i 2} T+\frac{a_{i 3}}{2} T^{2}+\frac{a_{i 4}}{3} T^{3}+\frac{a_{i 5}}{4} T^{4}+a_{i 7}\right),
$$

where, for each chemical species $i, a_{i j}$ are tabulated constants. 
In contrast, in the case of isobaric isothermal and isochoric isothermal reactors, the Lyapunov function is based on the mixture Gibbs free energy $\bar{g}$ and Helmholtz free en$\operatorname{ergy} \bar{f}$, respectively, as follows:

$$
\begin{aligned}
& G_{p, T}=\bar{g} / \mathcal{R} T=\bar{u} / \mathcal{R} T-\bar{s} / \mathcal{R}, \\
& G_{V, T}=\bar{f} / \mathcal{R} T=\bar{h} / \mathcal{R} T-\bar{s} / \mathcal{R} .
\end{aligned}
$$

A different form of a Lyapunov function for both the latter cases can be obtained from (4.4) and (4.2)

$$
G=\frac{1}{\bar{\rho}} \sum_{i=1}^{n}\left[c_{i} \ln \left(c_{i}\right)+\ln (\mathcal{R} T) c_{i}\right],
$$

where $\bar{\rho}=\sum_{i} c_{i} W_{i}$ is the mixture mean density, $W_{i}$ the molecular weight of species $i$, and all the constant terms, due to isothermal conditions, are neglected.

Here, it proves convenient, for various constructions discussed later on in the paper, to introduce the definition of entropic scalar product

$$
\langle x, y\rangle_{c}=(x, H y),
$$

where $\boldsymbol{H}=\left[\partial^{2} G / \partial c_{i} \partial c_{j}\right]$ is the second derivative matrix of $G$, while $\boldsymbol{x}$ and $\boldsymbol{y}$ are two arbitrary vectors. Note that the latter scalar product depends on the point $c$ in which the Hessian matrix $\boldsymbol{H}$ is evaluated. However, in the following, in order to save notation we shall use the symbol $\langle\boldsymbol{x}, \boldsymbol{y}\rangle$ instead of $\langle\boldsymbol{x}, \boldsymbol{y}\rangle_{c}$. Moreover, in the following, the linear functional appearing in the left-hand side of (4.1) will be denoted as

$$
D G(x)=(\nabla G, x),
$$

with $\operatorname{ker} D G$ indicating its null space.

\subsection{Thermodynamic projector}

In general, the invariance condition (2.3) is valid for an arbitrary projector $\boldsymbol{P}$, whereas the tangent space $T_{c}$, computed at the SIM point $c$, is spanned by the projector image:

$$
\operatorname{im}(\boldsymbol{P}(\boldsymbol{c})) \equiv T_{\boldsymbol{c}}
$$

However, generalizing notions of Section 2.2 leads to a special construction of $\boldsymbol{P}$, which has its own name: thermodynamic projector $[7,23,25]$. The idea behind the thermodynamic projector is the following: If a given manifold indeed represents the manifold of slow motions, then the Lyapunov function $G$ has been decreasing during the fast process of relaxation towards this manifold. Therefore, the points of the manifold appear as the minimum points of the Lypunov function on the manifolds of fast motion. The latter can be approximated accurately in a small vicinity of the slow manifold using the Lyapunov 
function gradient at the points of SIM. This intuitive picture is formalized below. Importantly, the thermodynamic projector is updated on each iterations when seeking the SIM from the invariance condition as illustrated below in Section 5.2.

Let us consider a grid approximating a $m$-dimensional slow invariant manifold. If $\mathcal{G}$ is a discrete subset in the $m$-dimensional parameter space $\boldsymbol{R}^{m}=\left\{\xi_{1}, \cdots, \xi_{m}\right\},\left.F\right|_{\mathcal{G}}$ denotes a discrete mapping of $\mathcal{G}$ into the concentration space. Let us consider an approximation procedure to restore the smooth map $F$ from the discrete one $\left.F\right|_{\mathcal{G}}$. The derivatives $f_{i}=$ $\partial F / \partial \xi_{i}$ are available, and the tangent space, at any grid point $c$, is

$$
T_{c}=\operatorname{Lin}\left\{f_{i}\right\}, \quad i=1, \cdots, m .
$$

The subspace $T_{c, 0}=\left(T_{\mathcal{c}} \cap \operatorname{ker} D G\right)$ defines, if $T_{\mathcal{c}} \neq T_{\boldsymbol{c}, 0}$, the tangent vector $\boldsymbol{e} \in T_{\mathcal{c}}$, through the following conditions

$$
\begin{aligned}
& \langle\boldsymbol{e}, \boldsymbol{x}\rangle=0, \quad \forall \boldsymbol{x} \in T_{\boldsymbol{c}, 0}, \\
& D G(\boldsymbol{e})=1,
\end{aligned}
$$

so that, the thermodynamic projection of an arbitrary vector $x$ has the form

$$
\boldsymbol{P} \boldsymbol{x}=D G(x) \boldsymbol{e}+\sum_{i=1}^{m-1}\left\langle\boldsymbol{k}_{i}, \boldsymbol{x}\right\rangle \boldsymbol{k}_{i}
$$

The basis $\left\{\boldsymbol{k}_{1}, \cdots, \boldsymbol{k}_{m-1}\right\}$ (orthonormal with respect to the entropic scalar product (4.6)) spans the subspace $T_{c, 0}$. In the case $T_{c} \equiv T_{c, 0}$, the projector (4.10) becomes

$$
\boldsymbol{P} \boldsymbol{x}=\sum_{i=1}^{m}\left\langle\boldsymbol{k}_{i}, \boldsymbol{x}\right\rangle \boldsymbol{k}_{i}
$$

Remark 4.1. Here, it is worth stressing the relevant feature of the latter projector. Let us consider a $m$-dimensional SIM in a $n$-dimensional phase space. The above construction is based on the idea that the thermodynamic considerations (minimization of the thermodynamic Lyapunov function) are solely required to construct fast manifolds in the vicinity of SIM. On the other hand, if it is possible to describe the fast subspace in different terms (for example, as a result of a different algorithm for construction of SIM), both representations of fast motions should be consistent. Let a vector $\boldsymbol{a}_{r_{i}}(\boldsymbol{c})$ be a generic vector of the fast subspace. Then

$$
\boldsymbol{a}_{r_{i}}(\boldsymbol{c}) \in \operatorname{ker} \boldsymbol{P}, \quad \forall i=1, \cdots, n-m,
$$

where $\operatorname{ker} \boldsymbol{P}$ is the null space of (4.10) evaluated at $c$. In other words, the thermodynamic projection of fast directions, in a neighborhood of the SIM, is "almost" null. We shall illustrate that the consistency is indeed achieved by considering a numerical example below where the fast manifold is defined as the fast subspace of the Jacobian matrix at the points of a SIM. 


\section{Method of invariant grids}

In this section, we present a general overview of the Method of Invariant Grids (MIG). The interested reader can find more details in $[6,7,16-20]$.

\subsection{Slow invariant grids}

For computational purposes, the discrete analogue of the problem of slow invariant manifold was developed in [6,7].

We are looking for SIM as an immersion $F$ of the parameter domain $\mathcal{W}$ into $\mathcal{D}, F: \mathcal{W} \rightarrow$ $\mathcal{D}$. Now we consider a discrete subset $\mathcal{G} \subset \mathcal{W}$. All functions are given on $\mathcal{G}$, and their smooth continuation on $\mathcal{W}$ could be constructed by various approximation technique (e.g., by low-order interpolation). We use notation $\left.F\right|_{\mathcal{G}}$ for restricting a function on a grid. An approximation technique gives a smooth function $F\left[\left.F\right|_{\mathcal{G}}\right]$. Let the transformation of a discrete set of values into a smooth function

$$
\left.F\right|_{\mathcal{G}} \longmapsto F\left[\left.F\right|_{\mathcal{G}}\right],
$$

be chosen. For each $y \in \mathcal{G}$ image of the differential $D_{y} F\left[\left.F\right|_{\mathcal{G}}\right](y)$ is a "tangent plain" to discrete set $\left.F\right|_{\mathcal{G}}\left(\mathcal{G}\right.$ at point $\left.\left.F\right|_{\mathcal{G}}(y)\right)$ :

$$
T_{y}=\operatorname{im} D_{y} F\left[\left.F\right|_{\mathcal{G}}\right](y) .
$$

We call $\left.F\right|_{\mathcal{G}}(\mathcal{G})$ an invariant grid, if it satisfies the grid version of the invariance equation

$$
(1-\boldsymbol{P}) \boldsymbol{J}(F(y))=0, \quad \text { for } y \in \mathcal{G}, \boldsymbol{P}: \boldsymbol{R}^{n} \rightarrow T_{y} .
$$

The grid version of the film equation (2.4) is also a motion in the defect of invariance direction $(1-\boldsymbol{P}) \boldsymbol{J}(F(y))$. The Newton method with incomplete linearization for grids has the same form as for continuous manifolds, and it is illustrated in Section 5.2. Hence, we can define the slow invariant grid as a stable fixed point of (one of) these processes. Formula (2.6) gives a velocity vector field on $\mathcal{G}$ and the approximation methods continue this vector field on the parameter space $\mathcal{W}$, as illustrated in more detail in Section 7 . This is the reduced model.

\subsection{Grid refinements}

Using grid connectivity, one can introduce differentiation operators in order to estimate the tangent vectors and define the projector operator $\boldsymbol{P}$ at every node. Let us assume that a rough approximation of the slow invariant manifold $\Omega$ can be constructed, and let $\mathcal{G}_{0}$ be a grid describing this approximation. In general, the invariance defect $\Delta$ is not expected to vanish at each node of $\mathcal{G}_{0}$, and the latter grid has to be refined. Namely, a generic grid node $c$ can be updated and shifted to the new location, $c+\delta c$, with a smaller defect of invariance

$$
\Delta=[1-P] J(c+\delta c)
$$


The node correction $\delta c$ can be computed by solving the incompletely linearized invariance condition [24] where the vector field $J$ is expanded up to the first order about $c$, while the projector $\boldsymbol{P}$ up to the zeroth order

$$
[1-\boldsymbol{P}(\boldsymbol{c})][\boldsymbol{J}(\boldsymbol{c})+\boldsymbol{L}(\boldsymbol{c}) \delta \boldsymbol{c}]=0,
$$

with $L=\left[\partial J_{i} / \partial c_{j}\right]$ denoting the first derivative matrix of the vector field $J$.

Grid refinements can be accomplished using the Newton method with incomplete linearization supplemented with the solvability condition (i.e., corrections are movements in the fast subspace)

$$
\boldsymbol{P} \delta \boldsymbol{c}=0 .
$$

Let us denote the null space of $\boldsymbol{D}$ and $\boldsymbol{P}$ as $\operatorname{ker} \boldsymbol{D}$ and $\operatorname{ker} \boldsymbol{P}$, respectively. It proves convenient to choose an orthonormal basis $\left\{\boldsymbol{b}_{i}\right\}$ in the subspace

$$
\boldsymbol{S}=(\operatorname{ker} \boldsymbol{D} \cap \operatorname{ker} \boldsymbol{P}),
$$

with respect to a proper scalar product. Let the dimension of $S$ be $r=\operatorname{dim}(S)$, the correction can be cast in the following form

$$
\delta \boldsymbol{c}=\sum_{i=1}^{r} \delta_{i} \boldsymbol{b}_{i}
$$

so that the linearized invariance equation (5.2) becomes a linear algebraic system in terms of $\delta_{i}$

$$
\sum_{i=1}^{r} \delta_{i}\left((1-\boldsymbol{P}) \boldsymbol{L} \boldsymbol{b}_{i}, \boldsymbol{b}_{k}\right)=-\left((1-\boldsymbol{P}) \boldsymbol{J}, \boldsymbol{b}_{k}\right), \quad k=1, \cdots, r,
$$

where the notation $(x, y)$ indicates the Euclidean scalar product of two arbitrary vectors $x$ and $y$.

Remark 5.1. The usual scalar product (,) was used to compute the components of the left-hand side of (5.2) with respect to the basis vectors $\left\{\boldsymbol{b}_{i}\right\}$. Nevertheless, a different scalar product can be also used without losing generality.

In the case of the thermodynamic projector (4.10), it proves convenient to choose the basis $\left\{\boldsymbol{b}_{i}\right\}$ orthonormal with respect to the entropic scalar product $\langle$,$\rangle (see Sections 4.1$ and 4.2) and write (5.4) as follows:

$$
\sum_{i=1}^{h} \delta_{i}\left\langle(1-\boldsymbol{P}) \boldsymbol{L} \boldsymbol{b}_{i}, \boldsymbol{b}_{k}\right\rangle=-\left\langle(1-\boldsymbol{P}) \boldsymbol{J}, \boldsymbol{b}_{k}\right\rangle, \quad k=1, \cdots, h .
$$

In this case, since (4.10) is "almost" $\langle$,$\rangle -orthogonal (\langle\operatorname{im} \boldsymbol{P}, \operatorname{ker} \boldsymbol{P}\rangle \cong 0)$ in a neighborhood of the SIM, (5.5) can be approximated and simplified as follows:

$$
\sum_{i=1}^{h} \delta_{i}\left\langle\boldsymbol{L} \boldsymbol{b}_{i}, \boldsymbol{b}_{k}\right\rangle=-\left\langle\boldsymbol{J}, \boldsymbol{b}_{k}\right\rangle, \quad k=1, \cdots, h .
$$


In contrast, according to the relaxation method (due to film equation (2.4)), the node correction takes on the form

$$
\delta c=\tau \Delta(c),
$$

where the parameter $\tau$ has the dimension of time. Estimates of $\tau$ can be found in the literature [7]

$$
\tau=-\frac{\langle\Delta, \Delta\rangle}{\langle\Delta, L \Delta\rangle}
$$

Notice however that, both the Newton method and the relaxation method may face the problem of the Courant instability in the case of fine grids, as illustrated in more detail in Section 5.5 and $[7,16]$.

\subsection{Initial grid based on quasi-equilibrium manifolds}

As demonstrated in [16], various approximated descriptions of SIM can be utilized in order to initialize MIG iterations. A cheap and accurate enough approximation is provided by quasi-equilibrium manifolds (QEM). By a definition, a $q$-dimensional QEM is a manifold in the concentration space which minimizes a Lyapunov function $G$ (with respect to the kinetic equation) under a set of $q$ linear constraints. Let us consider the minimization problem

$$
\left\{\begin{array}{l}
G(\boldsymbol{c}) \rightarrow \min , \\
\sum_{i=1}^{n} a_{i}^{j} c_{i}=\xi_{j}, \quad j=1, \cdots, q,
\end{array}\right.
$$

where $\left\{\boldsymbol{a}^{j}=\left(a_{1}^{j}, \cdots, a_{n}^{j}\right)\right\}$ is a set of $q$ fixed vectors expressing the primitive variables $c_{i}$ in terms of new parameters $\xi_{j}$. Because of convexity of $G$, once $q$ values are assigned to the quantities $\xi_{j}$, the solution of (5.8) is unique when it exists [40]. Regarding $\xi_{j}$ as variables, the joint set of constrained minima forms the $q$-dimensional quasi-equilibrium manifold (QEM) corresponding to the vector set $\left\{\boldsymbol{a}^{j}\right\}$. Quasi-equilibrium manifolds provide reasonably good initial approximations of slow invariant manifolds, and the rationale behind is discussed in Section 2.2. Moreover, comparisons between QEM and SIM can be also found in the literature $[16,18]$.

Before proceeding further, we note that the QEM can be by itself of interest for model reduction even before any refinement is addressed. For instance, the rate-controlled constrained-equilibrium (RCCE) method uses directly the notion of QEM for simplifying reaction mechanisms in combustion [41-43].

For the sake of completeness, below we briefly review the quasi-equilibrium grid algorithm [18] for constructing a grid based approximation of a QEM. Let $E$ be the $(d+$ $q) \times n$ matrix, constructed by adding the $\boldsymbol{a}^{j}$ vectors as $q$ additional rows to the matrix $\boldsymbol{D}$. Let the steady state of (3.6) be denoted by

$$
c^{0}=\left(c_{1}^{0}, \cdots, c_{n}^{0}\right),
$$


a QEM state $c^{1}$ can be computed, in a neighborhood of $c^{0}$, by solving the linear algebraic system

$$
\begin{aligned}
& \sum_{i=1}^{z}\left\langle\boldsymbol{t}_{j}, \boldsymbol{\rho}_{i}\right\rangle \varphi_{i}=-\left(\boldsymbol{\nabla} G, \boldsymbol{t}_{j}\right), \quad j=1, \cdots, z-q, \\
& \sum_{i=1}^{z}\left(\boldsymbol{a}^{1}, \boldsymbol{\rho}_{i}\right) \varphi_{i}=0, \cdots, \sum_{i=1}^{z}\left(\boldsymbol{a}^{k}, \boldsymbol{\rho}_{i}\right) \varphi_{i}=\varepsilon_{k}, \cdots, \sum_{i=1}^{z}\left(\boldsymbol{a}^{q}, \boldsymbol{\rho}_{i}\right) \varphi_{i}=0,
\end{aligned}
$$

with respect to the unknowns $\varphi_{i}$. If $\left\{\boldsymbol{\rho}_{1}, \cdots, \boldsymbol{\rho}_{z}\right\}$ and $\left\{\boldsymbol{t}_{1}, \cdots, \boldsymbol{t}_{z-q}\right\}$ are two vector bases spanning the null space of the matrix $D$ and $E$, respectively, then

$$
\begin{aligned}
& c^{1}=\left(c_{1}^{0}+d c_{1}, \cdots, c_{n}^{0}+d c_{n}\right), \\
& \left(d c_{1}, \cdots, d c_{n}\right)=\sum_{i=1}^{z} \varphi_{i} \boldsymbol{\rho}_{i} .
\end{aligned}
$$

By referring to system (5.9), all derivatives of $G$ are evaluated at $c^{0}$ and, through the last $q$ equations, we impose that $c^{1}$ belongs to a Cartesian grid in the space $\left\{\xi^{1}, \cdots, \xi^{q}\right\}$, with the fixed parameter $\varepsilon_{k}$ defining the grid step along $\xi^{k}$. Similarly, by solving (5.9) at $c^{1}$, a new QEM point $c^{2}$ can be found. In general, this procedure can be iterated as long as all the coordinates of the computed state are non-negative. The collection of computed states is called the quasi-equilibrium grid.

Finally, in the literature there are suggestions for choosing the set of parameterization vectors $\left\{\boldsymbol{a}^{j}\right\}$. For instance, the spectral quasi-equilibrium manifold is constructed when those vectors are given by $q$ left eigenvectors of the Jacobian matrix $L$ at the steady state, corresponding to the $q$ smallest eigenvalues by absolute value [16]. As an alternative, the parameters $\xi_{j}$ in (5.8) may represent physical quantities such as total number of moles, active valence or free oxygen [42].

\subsection{Construction under non-isothermal conditions}

In the case of non-isothermal conditions, an additional equation is needed in order to close the system of kinetic equations (3.2). This equation imposes the conservation of internal energy $\bar{u}$ or enthalpy $\bar{h}$, thus, for isolated and isobaric isenthalpic reactors, the following quantities

$$
\bar{u}=\sum_{i=1}^{n} u_{i}(T) Y_{i}, \quad \bar{h}=\sum_{i=1}^{n} h_{i}(T) Y_{i}
$$

are fixed, respectively. In the following, we deal with ideal gas mixtures where the internal energy $u_{i}$ and enthalpy $h_{i}$ of species $i$ are assumed to depend only on the temperature, while the corresponding mass fraction is denoted by $Y_{i}$. Moreover, polynomial fits 
are typically utilized to account for the temperature dependence [26] of $u_{i}(T)$ and $h_{i}(T)$ :

$$
\begin{aligned}
& u_{i}(T)=\mathcal{R}\left(a_{i 1} T+\frac{a_{2 i}}{2} T^{2}+\frac{a_{3 i}}{3} T^{3}+\frac{a_{4 i}}{4} T^{4}+\frac{a_{5 i}}{5} T^{5}+a_{6 i}\right)-\mathcal{R} T, \\
& h_{i}(T)=u_{i}(T)+\mathcal{R} T .
\end{aligned}
$$

A Lyapunov function of the kinetic system (3.2) can take the more general expression

$$
\tilde{G}=G+\sum_{i=1}^{l} \lambda_{i} \sum_{j=1}^{n} d_{i j} c_{j},
$$

where the $G$ function is defined as illustrated in Section $4.1, d_{i j}$ is a generic element of the matrix $\boldsymbol{D}$ defined in (3.7), and the fixed quantities $\lambda_{i}$ are determined imposing that, at the steady state $c^{e q}$, the gradient

$$
\nabla \tilde{G}\left(c^{e q}\right)=\mathbf{0} .
$$

By observing that the rightmost term of (5.13) is a linear combination of conserved quantities, it is straightforward to prove that $\tilde{G}$ represents a Lyapunov function with respect to the kinetic equation (3.2), as soon as the function $G$ also does. When dealing with nonisothermal reactors, it proves convenient to choose the function $\tilde{G}$ in order to construct the thermodynamic projector (4.10), and implement the numerical procedures described in Sections 5.2 and 5.3. Indeed, if all derivatives $\nabla \tilde{G}$ and $\boldsymbol{H}$ are computed by differentiating (5.13) under fixed energy $\bar{u}$ (or enthalpy $\bar{h}$ ), then both the conservation of atoms (3.7) and Eqs. (5.11) are automatically taken into account. In this case, we do not need to include any further equation to describe the temperature changes in the reaction: temperature values at any node of the invariant grid can be evaluated afterwards, upon solution of (5.12) by Newton-Raphson method. Exact expressions for computing first and second derivatives of $\tilde{G}$ can be found in [21].

\subsection{Uniqueness, stability and computational effort}

In general, there are many solutions to the invariance condition (2.3) which could be considered as a candidate of slow invariant manifolds. For instance, a subset of those solutions is given by all semi-trajectories $\{c(t), t>0\}$ which are obviously invariant. Moreover, in a vicinity of a stable equilibrium point, almost all solutions have the slowest Lyapunov exponent. Hence, almost all semi-trajectories could be considered as onedimensional slow invariant manifolds, since they meet intuitive expectations asymptotically at $t \rightarrow+\infty$. It becomes necessary to select one slow manifold that is the best, in some sense. It can be proven [17] that definition of slow invariant manifolds as the stable fixed point of iterative procedure described in Section 2.2 reduces significantly the set of solutions to (2.3) and, in a neighborhood of a stable equilibrium of an analytical system, it guarantees uniqueness of analytical slow invariant manifolds.

Based on our numerical computations, constructing invariant grids by the above iterative methods has never led to multiple solutions. In this respect, it is also important 
to reflect on the consequences of a possible convergence of the proposed numerical algorithm to an invariant solution which is not slow, say a generic semi-trajectory. In this case, though one would expect that corrections (5.4), (5.6) and (5.7) vanish, they do not. An arbitrary semi-trajectory is invariant under the dynamics of the kinetic system (3.2), but not stable under the iterative refinement of Section 5.2. Indeed, in this case, small perturbations (always present in any calculation on computers) have the effect to make non-invariant the solution to the next iteration. Several illustrative examples, where the initial grid was chosen in the vicinity of solution trajectories, confirmed the above feature of the MIG algorithm. Once again, we intend to stress the crucial result that iteration stability represents the key point for slowness.

Notice that, although rigorous proof concerning the existence of solutions to the Newton method with incomplete linearization (see Sections 2.2 and 5.2) for non-linear systems are not yet available in the literature, rigorous proofs of global existence and uniqueness of slow invariant manifolds, obtained by the film equation of dynamics (2.4), were obtained recently for a class of linear problems [15].

Both the Newton method with incomplete linearization and the relaxation method (Section 5.2) are explicit numerical schemes to solve the invariance condition (2.3) and the film equation (2.4), respectively. Therefore, as it was first pointed out in [7], they may face the issue of Courant instability. For instance, if the initial grid is characterized by a small node spacing, at some nodes a norm of the defect of invariance $\Delta$ exhibits a divergent behavior, instead of dying out as iterations proceed further (see also $[7,16]$ ). A way to avoid such instability is well known. This is decreasing the time step. In our problem, instead of a true time step, we have a shift in the Newtonian direction. Formally, we can assign the value $\tilde{h}=1$ for one complete step in the Newtonian direction, so that we can extend the Newton method to arbitrary $\tilde{h}$. To this end, let us find $\delta_{i}$, e.g., from (5.4), and update $\delta c$ proportionally to $\tilde{h}$; the corrected node is

$$
c_{n+1}=c_{n}+\tilde{h} \delta c_{n}
$$

where the subscript $n$ denotes the number of iteration. Everywhere the parameter $\tilde{h}$ is maintained as large as possible without running into convergence problems. In the following, if not otherwise stated, we use $\tilde{h}=1$. Similar considerations are applied to eliminate the Courant instability in the case of relaxation method.

Finally, it is worth pointing out that the number of nodes of uniform grids (therefore the computational effort associated with the MIG approach, as well as with any other grid-based method for model reduction) scales as $\varepsilon^{-q}$, where $q$ is the grid dimension while the small parameter $\varepsilon$ is representative of the grid spacing. Though this may seem inefficient, we should stress that invariant grids are constructed and tabulated only once for a later and efficient integration of the kinetic equations. An estimate of the computational time needed for computing two-dimensional grids, in the case of realistic hydrogen combustion, is reported in [19]. 


\section{Illustration}

It is useful to explain the above notions with the help of two illustrative examples. First, we consider the four step three species reaction (Example 1):

$$
\begin{array}{ll}
\text { 1. } A \leftrightarrow B, & k_{1}^{+}=1, \\
\text { 2. } B \leftrightarrow C, & k_{2}^{+}=1, \\
\text { 3. } C \leftrightarrow A, & k_{3}^{+}=1, \\
\text { 4. } A+B \leftrightarrow 2 C, & k_{4}^{+}=50,
\end{array}
$$

in a closed system, under fixed volume and temperature. The atom balance reads

$$
c_{A}+c_{B}+c_{C}=1
$$

while the equilibrium point is chosen as $c_{A}^{e q}=0.1, c_{B}^{e q}=0.5, c_{C}^{e q}=0.4$. The inverse rate constants $k_{i}^{-}$can be calculated from the steady state and $k_{i}^{+}$, by invoking the principle of detailed balance (3.5). The latter mechanism is effectively two dimensional, and a 1dimensional slow invariant manifold can be constructed for its reduced description. For that reaction, the system (3.6) takes the explicit form

$$
\dot{\boldsymbol{c}}=\boldsymbol{J}(\boldsymbol{c})=W_{1}\left[\begin{array}{c}
-1 \\
1 \\
0
\end{array}\right]+W_{2}\left[\begin{array}{c}
0 \\
-1 \\
1
\end{array}\right]+W_{3}\left[\begin{array}{c}
1 \\
0 \\
-1
\end{array}\right]+W_{4}\left[\begin{array}{c}
-1 \\
-1 \\
2
\end{array}\right]
$$

where

$$
\begin{array}{ll}
W_{1}=k_{1}^{+} c_{A}-k_{1}^{-} c_{B}, & W_{2}=k_{2}^{+} c_{B}-k_{2}^{-} c_{C}, \\
W_{3}=k_{3}^{+} c_{C}-k_{3}^{-} c_{A}, & W_{4}=k_{4}^{+} c_{A} c_{B}-k_{4}^{-} c_{C}^{2} .
\end{array}
$$

Example 2 is a model for hydrogen oxidation, see, e.g., [17, p. 291]. Let us consider a closed system with six species $\mathrm{H}_{2}$ (hydrogen), $\mathrm{O}_{2}$ (oxygen), $\mathrm{H}_{2} \mathrm{O}$ (water), $\mathrm{H}, \mathrm{O}, \mathrm{OH}$ (radicals) reacting in a constant volume and under constant temperature. The detailed kinetic mechanism and the direct rate constants of each step are:
1. $H_{2} \leftrightarrow 2 H$,
$k_{1}^{+}=2$,
2. $\mathrm{O}_{2} \leftrightarrow 2 \mathrm{O}$,
$k_{2}^{+}=1$,
3. $\mathrm{H}_{2} \mathrm{O} \leftrightarrow \mathrm{H}+\mathrm{OH}, \quad k_{3}^{+}=1$,
4. $\mathrm{H}_{2}+\mathrm{O} \leftrightarrow \mathrm{H}+\mathrm{OH}, k_{4}^{+}=10^{3}$,
5. $\mathrm{O}_{2}+\mathrm{H} \leftrightarrow \mathrm{O}+\mathrm{OH}, \quad k_{5}^{+}=10^{3}$,
6. $\mathrm{H}_{2}+\mathrm{O} \leftrightarrow \mathrm{H}_{2} \mathrm{O}, \quad k_{6}^{+}=10^{2}$. 
The atom balances read

$$
\begin{aligned}
& 2 c_{\mathrm{H}_{2}}+2 c_{\mathrm{H}_{2} \mathrm{O}}+c_{\mathrm{H}}+c_{\mathrm{OH}}=b_{\mathrm{H}}=2, \\
& 2 c_{\mathrm{O}_{2}}+c_{\mathrm{H}_{2} \mathrm{O}}+c_{\mathrm{O}}+c_{\mathrm{OH}}=b_{\mathrm{O}}=1,
\end{aligned}
$$

and the equilibrium point possesses now the following coordinates:

$$
\begin{array}{lll}
c_{\mathrm{H}_{2}}^{e q}=0.27, & c_{\mathrm{O}_{2}}^{e q}=0.135, & c_{\mathrm{H}_{2} \mathrm{O}}^{e q}=0.7, \\
c_{\mathrm{H}}^{e q}=0.05, & c_{\mathrm{O}}^{e q}=0.02, & c_{\mathrm{OH}}^{e q}=0.01 .
\end{array}
$$

The concentration space is 6-dimensional, but the effective dimension is four because of the linear constraints (6.5). This time, both 1- and 2-dimensional slow invariant manifolds can be constructed. Time evolution of molar concentrations, as dictated by (6.4), is described by the following system of equations

$$
\dot{\boldsymbol{c}}=\boldsymbol{J}(\boldsymbol{c})=\left[\begin{array}{c}
-W_{1}^{\prime}-W_{4}^{\prime}-W_{6}^{\prime} \\
-W_{2}^{\prime}-W_{5}^{\prime} \\
-W_{3}^{\prime}+W_{6}^{\prime} \\
2 W_{1}^{\prime}+W_{3}^{\prime}+W_{4}^{\prime}-W_{5}^{\prime} \\
2 W_{2}^{\prime}-W_{4}^{\prime}+W_{5}^{\prime}-W_{6}^{\prime} \\
W_{3}^{\prime}+W_{4}^{\prime}+W_{5}^{\prime}
\end{array}\right]
$$

where

$$
\begin{array}{ll}
W_{1}^{\prime}=k_{1}^{+} c_{\mathrm{H}_{2}}-k_{1}^{-} c_{H}^{2}, & W_{2}^{\prime}=k_{2}^{+} c_{\mathrm{O}_{2}}-k_{2}^{-} c_{\mathrm{O}}^{2}, \\
W_{3}^{\prime}=k_{3}^{+} c_{\mathrm{H}_{2} \mathrm{O}}-k_{3}^{-} c_{H} c_{\mathrm{OH}}, & W_{4}^{\prime}=k_{4}^{+} c_{\mathrm{H}_{2}} c_{\mathrm{O}}-k_{4}^{-} c_{H} c_{\mathrm{OH}}, \\
W_{5}^{\prime}=k_{5}^{+} c_{\mathrm{O}_{2}} c_{\mathrm{H}}-k_{5}^{-} c_{\mathrm{O}} c_{\mathrm{OH}}, & W_{6}^{\prime}=k_{6}^{+} c_{\mathrm{H}_{2}} c_{\mathrm{O}}-k_{6}^{-} c_{\mathrm{H}_{2} \mathrm{O} .}
\end{array}
$$

\subsection{Slow-fast motion decomposition}

In this section, we demonstrate that the construction of the thermodynamic projector performs decomposition of fast-slow motions of the kinetic equation (3.6). For the dynamical systems (6.2) and (6.6), the thermodynamic Lyapunov function is (4.5) which can be conveniently recast as:

$$
G=\sum_{i=1}^{n} c_{i}\left[\ln \left(c_{i} / c_{i}^{e q}\right)-1\right]
$$

where the dependence on the steady state coordinates is explicitly shown. Hence, the thermodynamic projector (4.10) takes, in the case of Example 1, the form

$$
\begin{aligned}
& \boldsymbol{P} \boldsymbol{x}=(\boldsymbol{\nabla} G, \boldsymbol{x}) \boldsymbol{e} \\
& \boldsymbol{\nabla} G=\left(\ln \left(c_{A} / c_{A}^{e q}\right), \ln \left(c_{B} / c_{B}^{e q}\right), \ln \left(c_{C} / c_{C}^{e q}\right)\right) .
\end{aligned}
$$



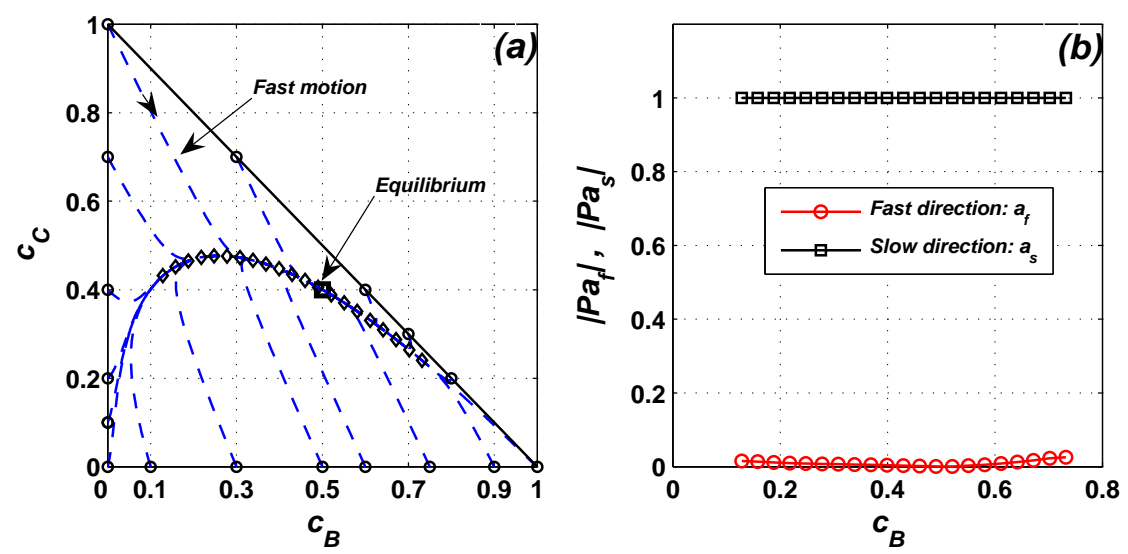

Figure 2: (Color online). Four step three species mechanism. (a) Some solution trajectories of the system (6.2) in the phase-space $c_{B}-c_{C}$ (dashed lines). Invariant grid (diamonds). (b) Euclidean norm of the thermodynamic projection of Jacobian eigenvectors in the grid nodes.

Fig. 2(a) shows solution trajectory samples of the kinetic equation (6.2) in the plane $c_{B}-c_{C}$. In this case, a 1-dimensional invariant grid was constructed by refinements of a spectral quasi-equilibrium grid (see Section 5.3) using the Eqs. (5.4). At each node $c^{*}$ of the invariant grid, the Jacobian matrix $L\left(c^{*}\right)$ of (6.2) was considered, in order to estimate both the fast direction $a_{f}$ (eigenvector corresponding to the largest eigenvalue by absolute value) and the slow direction $a_{s}$ (eigenvector corresponding to the smallest eigenvalue by absolute value). In fact, it is known from the literature [27] that spectral decomposition of $\boldsymbol{L}$ enables to compute approximated slow and fast components of the vector of motion $J$.

Those eigenvectors were projected onto the tangent space of the invariant grid, by means of (6.9a), and in Fig. 2(b) the Euclidean norm $\left\|\boldsymbol{P} \boldsymbol{a}_{i}\right\|$ is plotted versus the grid parameter $c_{B}$, where the eigenvectors were chosen such that $\left\|a_{f}\right\|=\left\|a_{s}\right\|=1$. As shown in Fig. 3, the fast component of any trajectory lies in the affine subspace $c^{*}+\operatorname{ker} \boldsymbol{P}$, where $c^{*}$ is an arbitrary point of the SIM. A two-dimensional grid, invariant with respect to (6.6), was also constructed by applying the MIG procedure in the form of Newton iterations starting from the pertinent spectral quasi-equilibrium grid (see Fig. 4). In the case of Example 2, the thermodynamic projector (4.10) reads

$$
P x=(\nabla G, x) e+\langle k, x\rangle k .
$$

Now, the spectral analysis of the Jacobian $L$, at each grid node, delivers four non-zero eigenvalues. Let the eigenvectors be denoted as $a_{f}^{1}, a_{f}^{2}, a_{s}^{1}, a_{s}^{2}$ where the corresponding eigenvalues are ordered such that

$$
\lambda_{f}^{1}<\lambda_{f}^{2}<\lambda_{s}^{1}<\lambda_{s}^{2} .
$$

Thermodynamic projections $\boldsymbol{P a}$ of all eigenvectors $\boldsymbol{a}$ (with $\|\boldsymbol{a}\|=1$ ) were evaluated at every node, and their Euclidean norms $\|\boldsymbol{P a}\|$ reported in Fig. 5. 


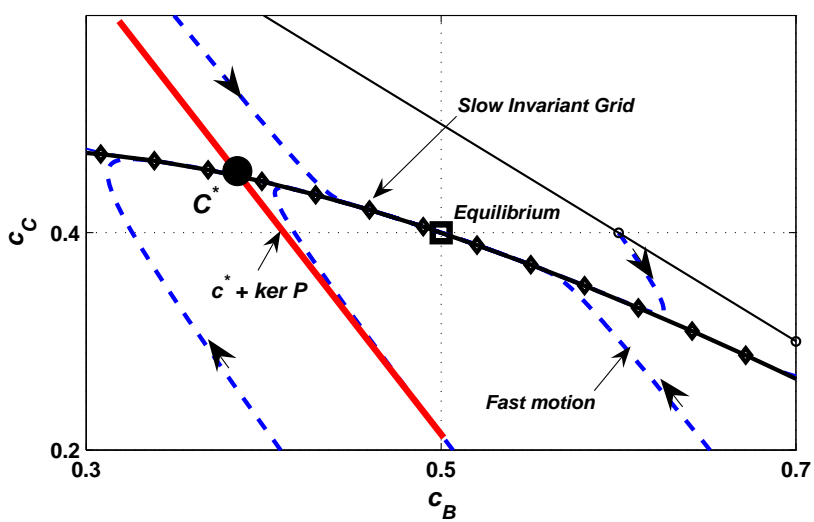

Figure 3: (Color online). Four step three species mechanism. The fast part of any trajectory belongs to the null space $(\operatorname{ker} \boldsymbol{P})$ of the thermodynamic projector $\boldsymbol{P}$.

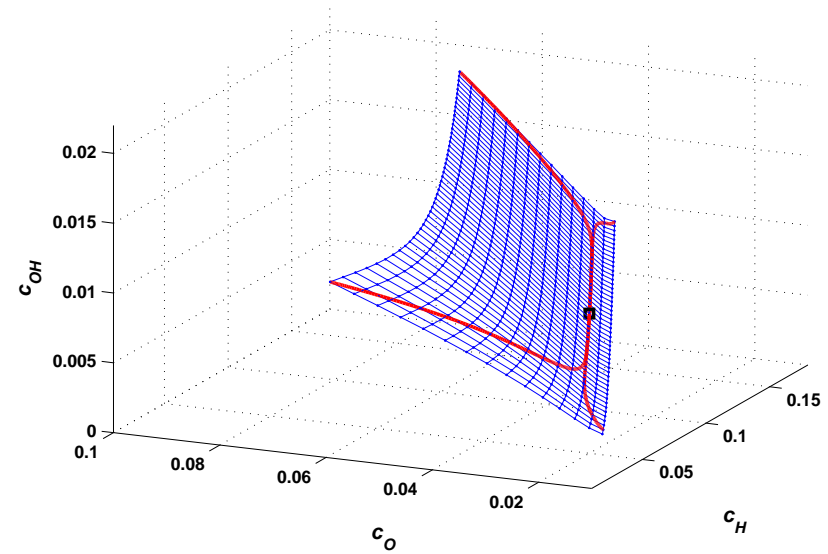

Figure 4: (Color online). Mechanism (6.4): Two dimensional invariant grid (dots). Solution trajectories of the system (6.6) (bold lines).
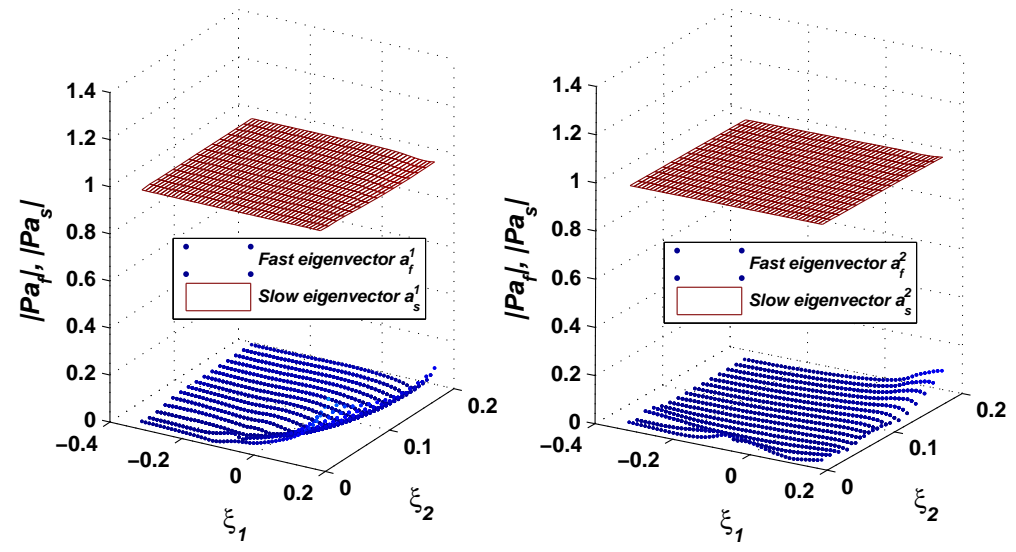

Figure 5: (Color online). Two dimensional invariant grid: Thermodynamic projection of fast and slow directions. A pair of parameters $\left(\xi_{1}, \xi_{2}\right)$ is uniquely associated with each grid point $c$. 
It is worth stressing that the fast projections do not completely vanish because of two reasons. First, the Jacobi matrix eigenvectors only represent an approximation of fast and slow directions. Second, as already mentioned in Section 2.2, the fast-slow motion decomposition based on thermodynamics is approximated. Agreement between two quite different pictures is nevertheless impressive.

\section{Access of grid nodes}

The described procedure delivers the location of the invariant grid nodes, and below we address the issue of how to utilize the computed set of states during the time integration of a reduced set of equations.

\subsection{Global parametrization}

Let $\Omega$ be the slow invariant manifold with respect to the system (3.6). Let $m$ and $n$ be the dimension of $\Omega$ and the phase space dimension, respectively. Here, we assume that $\Omega$ can be parameterized by a set of new coordinates

$$
y=\left[\xi_{1}, \cdots, \xi_{m}\right],
$$

obtained by the following linear transformation

$$
y=R c,
$$

where $\boldsymbol{R}$ is a fixed $m \times n$ matrix. By differentiating (7.1) with respect to time and invoking (2.5), the dynamics of (3.6) on the SIM can be written in terms of the new variables as

$$
\dot{y}=R \dot{c}=R P J .
$$

Since the right-hand side of the above formula depends on the primitive variables $c$, the latter system can be solved as soon as the explicit equation of the SIM is known

$$
c_{S I M}=\boldsymbol{c}\left(\xi_{1}, \cdots, \xi_{m}\right) .
$$

Let us assume that the MIG method delivers the invariant grid $\mathcal{G}$ approximating the slow invariant manifold $\Omega$. Using (7.1), a parameters set $\left(\xi_{1}^{c}, \cdots, \xi_{m}^{c}\right)$ can be associated to each node $c$ of $\mathcal{G}$. Although the analytical expression of the manifold equation is not known, the discrete mapping $c_{\mathcal{G}}\left(\xi_{1}^{c}, \cdots, \xi_{m}^{c}\right)$ is available, and interpolated values can be used when integrating the reduced system of equations: The implementation of this will be illustrated below with the help of Example 1 and Example 2.

Note that, the projector $\boldsymbol{P}$ of the reduced system (7.2) must be constructed as prescribed in Section 4.2. In this case, the fast directions belong to the null space of the thermodynamic projector, and the right-hand side of (7.2) is characterized by a reduced stiffness with respect to the original system (3.6) (see Section 6.1). 


\subsubsection{D grid based integrator: global parametrization}

Let the 1-dimensional invariant grid for the system (6.2) be stored in the three 1-dimensional arrays $p_{A}, p_{B}, p_{C}$ so that the $i$-th grid node has the following coordinates

$$
\boldsymbol{c}_{i}=\left[\boldsymbol{p}_{A}(i), \boldsymbol{p}_{B}(i), \boldsymbol{p}_{C}(i)\right] .
$$

Here, the choice of $\boldsymbol{R}$ is done according to the spectral quasi-equilibrium parametrization suggested in $[16,18]$. Therefore, $R$ denotes the left eigenvector, of the Jacobi matrix at the equilibrium, which corresponds to the smallest eigenvalue (by absolute value). Only one parameter

$$
\xi^{(i)}=\boldsymbol{R}\left[\begin{array}{l}
\boldsymbol{p}_{A}(i) \\
\boldsymbol{p}_{B}(i) \\
\boldsymbol{p}_{C}(i)
\end{array}\right], \quad \boldsymbol{R}=[0.881,-0.391,0.268],
$$

is now associated with each grid node $c_{i}$. Let parameters $\xi^{(i)}$ be stored in the 1-dimensional array

$$
\zeta=\left\{\tilde{\zeta}^{(i)}\right\}
$$

Here, the reduced system (7.2) takes the form

$$
\left\{\begin{array}{l}
\dot{\zeta}=R \boldsymbol{P J}(\boldsymbol{c}(\xi)) \\
\xi\left(t_{0}\right)=\xi_{0}
\end{array}\right.
$$

and the initial condition $\xi_{0}$ corresponds to the invariant grid node $c_{0}$. The case where $c_{0}$ does not belong to the grid will be presented in Section 8. Let the components of $\zeta$ strictly increase. If $Y$ denotes the number of grid nodes, for all parameters $\xi \in[\zeta(1), \zeta(\mathrm{Y})]$, a small interval $[\zeta(i), \zeta(i+v)]$ can be found such that $\xi$ belongs to it. By introducing the Lagrangian polynomials $l_{i}(x), \cdots, l_{i+v}(x)$ :

$$
l_{j}(x)=\prod_{k=i, k \neq j}^{i+v}(x-\zeta(k)) / \prod_{k=i, k \neq j}^{i+v}(\zeta(j)-\zeta(k)), \quad j=i, \cdots, i+v,
$$

the following primitive variables

$$
c_{A}=\sum_{j=i}^{i+v} \boldsymbol{p}_{A}(j) l_{j}(\xi), \quad c_{B}=\sum_{j=i}^{i+v} \boldsymbol{p}_{B}(j) l_{j}(\xi), \quad c_{C}=\sum_{j=i}^{i+v} \boldsymbol{p}_{C}(j) l_{j}(\xi),
$$

can be associated with the parameter $\xi$. Eq. (7.4) was numerically integrated using a fourth order Runge-Kutta explicit scheme where the right-hand side is evaluated through (7.6) with $v=2$. Fig. 6 shows a comparison between the full system and the reduced one. The time step for the reduced system was increased up to $\Delta t=0.5$ without a big loss of accuracy. Since the full system solution does not present significant deviations only when $\Delta t \leq 0.01$, we can argue that the reduced system is characterized by much less stiffness. 

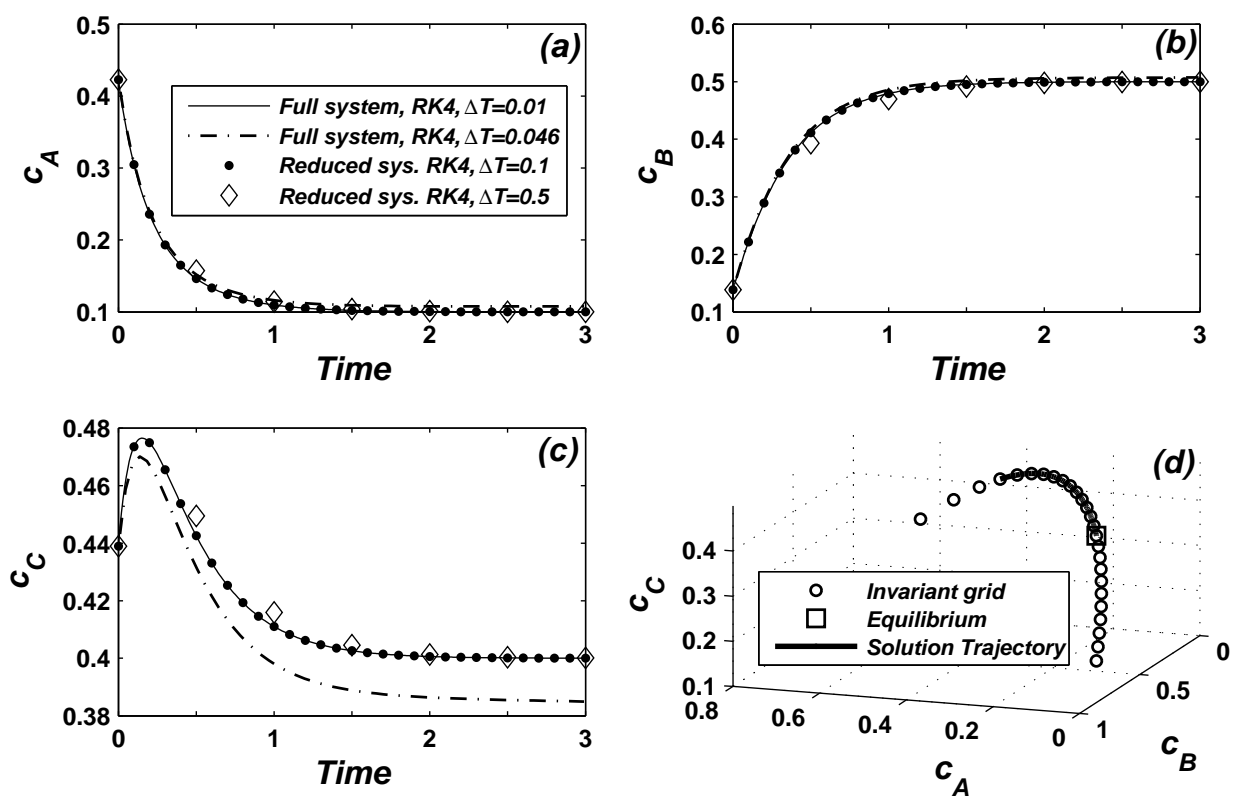

Figure 6: Time integration using a global parametrization of the invariant grid. An explicit fourth order RungeKutta scheme (RK4) was used for both (6.2) and (7.4), and a comparison with different time steps $\Delta t$ is reported. The initial condition was chosen as $\boldsymbol{c}_{0}=[0.423,0.138,0.439]$ so that $\xi_{0}=\boldsymbol{R} \boldsymbol{c}_{0}^{T}=0.436$.

\subsection{D grid based integrator: local parametrization}

The procedure described in Section 7.1.1 is based on the assumption that there exists a matrix $R$ such that the mapping (7.3) is unique ( $\zeta$ strictly monotonic). When this does not occur, the same value of $\xi$ is associated with different grid nodes: In this case, the global parametrization is not suitable for accessing the grid nodes during the integration of the reduced system (7.4). However, the above methodology can be regarded as a special case of a more general strategy. Indeed, in Section 7.1.1, the matrix $R$ is constructed by taking into account the slow directions at the equilibrium point $c^{e q}$ (quasi-equilibrium manifold parametrization). Therefore, $R$ is expected to provide the neighbors of the steady state with monotonic parameters, whereas this is not guaranteed when moving far from $c^{e q}$. In order to overcome this issue, the matrix $R$ can be dynamically updated during the time integration. Namely, $\boldsymbol{R}$ can be made dependent on the grid zone involved in the integration and, in the sequel, a possible way is presented.

Let the initial condition $c_{0}=\left[c_{A 0}, c_{B 0}, c_{C 0}\right]$ fulfill the following inequalities

$$
\left\{\begin{array}{l}
\boldsymbol{p}_{A}\left(i^{\prime}\right) \leq c_{A 0}<\boldsymbol{p}_{A}\left(i^{\prime}+v\right), \\
\boldsymbol{p}_{B}\left(i^{\prime}\right) \leq c_{B 0}<\boldsymbol{p}_{B}\left(i^{\prime}+v\right), \\
\boldsymbol{p}_{C}\left(i^{\prime}\right) \leq c_{C 0}<\boldsymbol{p}_{C}\left(i^{\prime}+v\right),
\end{array}\right.
$$



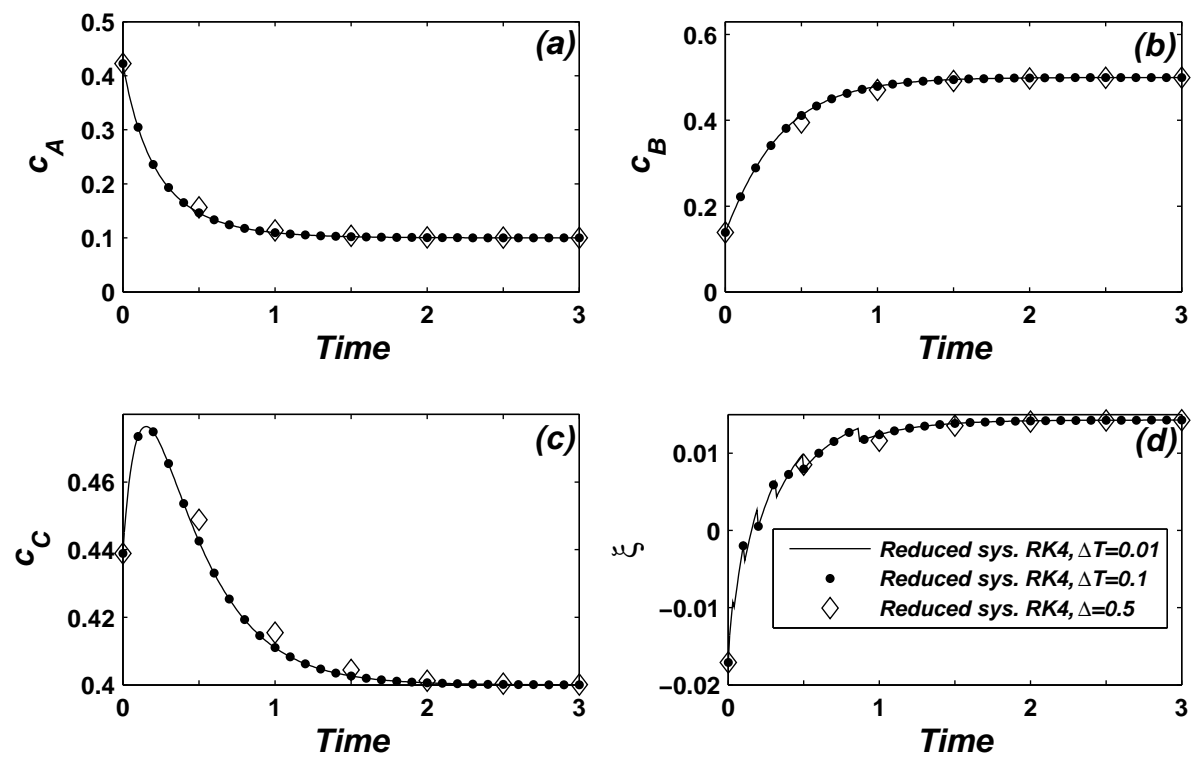

Figure 7: Time integration by using a local parametrization of the invariant grid. An explicit fourth order Runge-Kutta scheme (RK4) was used. The time steps $\Delta t$ was increased up to $\Delta t=0.5$, by starting from $c_{0}=[0.423,0.138,0.439]$.

then $R$ can be set as follows:

$$
\boldsymbol{R}_{i^{\prime}}=\left[\begin{array}{l}
\boldsymbol{p}_{A}\left(i^{\prime}+v\right)-\boldsymbol{p}_{A}\left(i^{\prime}\right) \\
\boldsymbol{p}_{B}\left(i^{\prime}+v\right)-\boldsymbol{p}_{B}\left(i^{\prime}\right) \\
\boldsymbol{p}_{C}\left(i^{\prime}+v\right)-\boldsymbol{p}_{C}\left(i^{\prime}\right)
\end{array}\right]^{T} .
$$

If $v$ is small enough, then $\boldsymbol{R}_{i^{\prime}}$ approximately spans the slow-subspace at the $i^{\prime}$-th node of the invariant grid. For that reason, the parametrization carried out through $\boldsymbol{R}_{i^{\prime}}$ is expected to associate uniquely the parameters with the grid nodes in a neighborhood of $c_{0}$. Starting from

$$
\xi_{0}=\boldsymbol{R}_{i^{\prime}} \boldsymbol{c}_{0}^{T},
$$

numerical integration of (7.4) can be performed, as described in Section 7.1.1, by using the vector $\boldsymbol{R}_{i^{\prime}}$ in order to evaluate the parameter $\xi_{1}$ after the first time step. For evaluating $\xi_{2}, \boldsymbol{R}_{i^{\prime}}$ is kept constant if $\xi_{1}$ still belongs to the interval $\left[\zeta\left(i^{\prime}\right), \zeta\left(i^{\prime}+v\right)\right]$; when that does not occur, the parametrization matrix is updated. In this case, a new index $i^{\prime \prime}$ is chosen, instead of $i^{\prime}$, such that the inequalities (7.7) hold for the point $c_{1}=c\left(\xi_{1}\right)$, and a new matrix $\boldsymbol{R}_{i^{\prime \prime}}$ introduced according to (7.8). Now, starting from $\boldsymbol{R}_{i^{\prime \prime}} \boldsymbol{c}_{1}^{T}$, the integration is carried on, and same procedure applies for subsequent time steps. Eq. (7.4) was solved by using an explicit fourth order Runge-Kutta scheme in combination with the local parametrization approach (see Fig. 7). The interpolation formulas (7.6) with $v=2$ were still adopted. Notice that, as shown in Fig. 7(d), the updating of the matrix $R$ makes the time evolution of the parameter $\xi$ non-smooth, although the dynamics is still smooth in terms of primitive variables. 


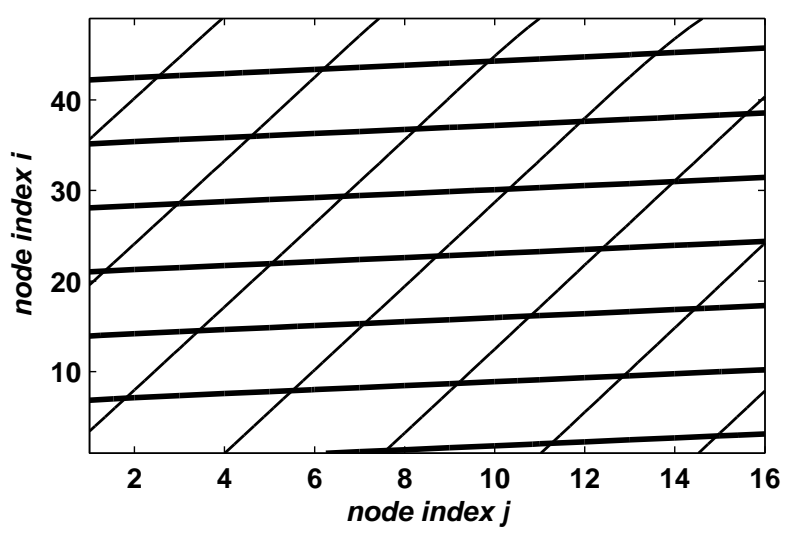

Figure 8: Level curves of the parameters $\xi$ (bold lines) and $\eta$ (thin lines). Two level lines have, if any, only one intersection point.

\subsubsection{D grid based integrator: global parametrization}

As described in Section 7.1, the two-dimensional grid of Fig. 4 can be globally parameterized through a fixed matrix $\boldsymbol{R}$. Here, the spectral quasi-equilibrium parametrization leads to the following $2 \times 6$ matrix

$$
\boldsymbol{R}=\left[\begin{array}{cccccc}
0.665 & 0.326 & -0.671 & -0.0103 & -0.00568 & 0.0228 \\
0.0450 & -0.0196 & 0.378 & -0.856 & -0.349 & 0.0103
\end{array}\right]
$$

It is worth stressing once again that $R$ is chosen in such a way that its rows are the slowest and the second slowest left eigenvector of the Jacobian matrix evaluated at the equilibrium point, respectively. Now, the invariant grid nodes can be ordered by means of two indexes. Each node $c$ is labeled by a pair $(i, j)$, so that the reduced variables of $c(i, j)$ are given by

$$
y^{(i, j)}=\left[\begin{array}{l}
\xi^{(i, j)} \\
\eta^{(i, j)}
\end{array}\right]=\boldsymbol{R} \boldsymbol{c}(i, j) .
$$

In this case, the matrix (7.9) delivers a unique mapping (7.10) (see Fig. 8), so that the reduced system (7.2) is now composed of the two following ordinary differential equations

$$
\left[\begin{array}{c}
\dot{\xi} \\
\dot{\eta}
\end{array}\right]=\operatorname{RPJ}(c(\xi, \eta))
$$

Since the explicit dependence of the vector field $J$ on the reduced parameters is not known, a proper interpolation of the discrete mapping (7.10) is needed and, in the sequel, a possible way to calculate the right-hand side of (7.11) is presented.

Let $\xi_{0}$ and $\eta_{0}$ be the initial condition for (7.11) whose location on the $(\xi, \eta)$-plane is denoted by a square in Fig. 9 . In this case, the point $c_{0}\left(\xi_{0}, \eta_{0}\right)$ can be reconstructed by the 


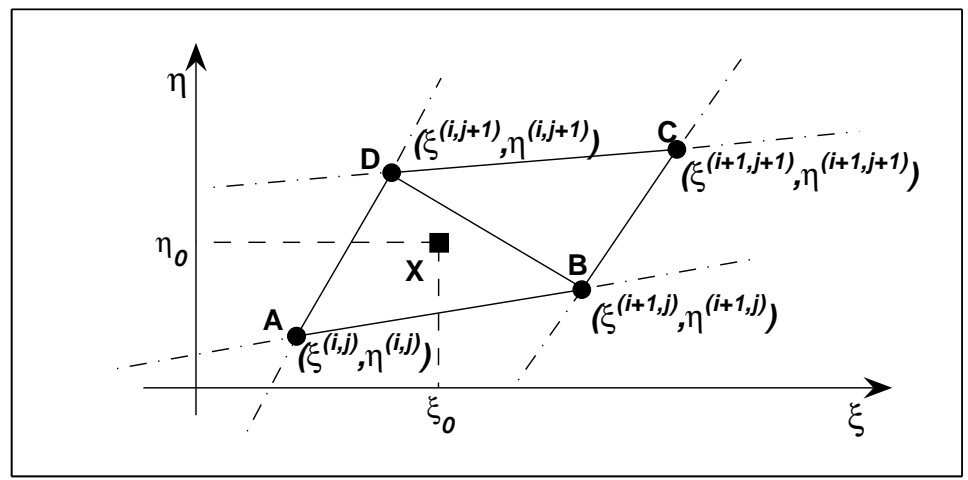

Figure 9: Reduced variables subspace $(\xi, \eta)$ : The linear transformation (7.10) maps the invariant grid nodes into a discrete set of points (circles).

following first-order interpolation scheme:

$$
\left\{\begin{array}{l}
\boldsymbol{c}_{0}\left(\xi_{0}, \eta_{0}\right)=w_{A} \boldsymbol{c}_{A}+w_{B} \boldsymbol{c}_{B}+w_{D} \boldsymbol{c}_{D} \\
w_{A}=\frac{A_{B D X}}{A_{A B D}}, \quad w_{B}=\frac{A_{A X D}}{A_{A B D}}, \quad w_{D}=\frac{A_{A B X}}{A_{A B D}}
\end{array}\right.
$$

where, e.g., $A_{A B D}$ represents the area of the triangle $A B D$. On the other hand, the latter point may be also attained by performing a bilinear interpolation over the four nodes reported in Fig. 9. The latter task is achieved by following a well known strategy used, e.g., in the finite element method (see, e.g., [38]). Namely, interpolation can be split in two steps. First of all, through a coordinate transformation (which maps an arbitrary foursided of Fig. 9 into the standard square centered in the origin whose edge length is 2 any pair $(\xi, \eta)$ can be transformed in the new one $(\bar{\xi}, \bar{\eta})$. Secondly, interpolation is performed on the standard square by using, for each corner, the corresponding Lagrangian weight

$$
\left\{\begin{array}{l}
w_{A}=(1-\bar{\xi})(1-\bar{\eta}) / 4, \\
w_{B}=(1+\bar{\zeta})(1-\bar{\eta}) / 4, \\
w_{C}=(1+\bar{\zeta})(1+\bar{\eta}) / 4, \\
w_{D}=(1-\bar{\xi})(1+\bar{\eta}) / 4,
\end{array}\right.
$$

so that the interpolated concentration vector

$$
\boldsymbol{c}(\xi, \eta)=w_{A} \boldsymbol{c}_{A}+w_{B} \boldsymbol{c}_{B}+w_{C} \boldsymbol{c}_{C}+w_{D} \boldsymbol{c}_{D},
$$

enables to evaluate the right-hand side of (7.11) at each time step. A comparison between the numerical solution of the full system (6.6) and the reduced one (7.11) is reported in Fig. 10. Here, the bilinear interpolation was performed. The former system can be accurately integrated choosing the time step not larger than $\Delta t \approx 7 \times 10^{-4}$ (explicit RK4 scheme); while (7.11) was integrated by increasing $\Delta t$ up to $2 \times 10^{-2}$. Moreover, (7.14) can be generalized in order to use higher order interpolation schemes. In that case, all the grid nodes with labels $(i+l, j+\kappa) l, \kappa=0, \cdots, v$, where $v$ denotes the polynomial order, need to be involved: For instance, the Fig. 9 shows the case with $v=1$. 

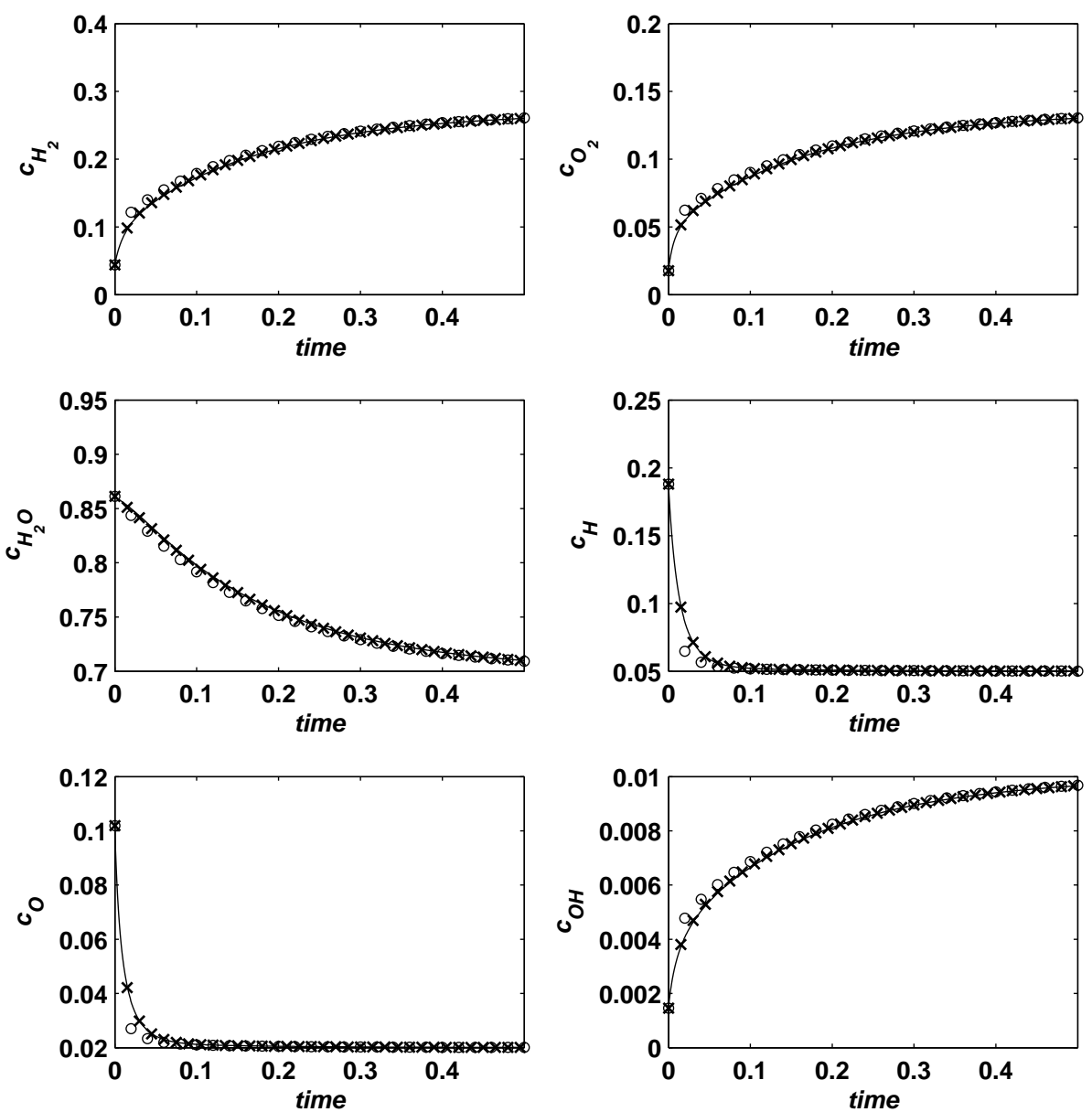

Figure 10: Global and local parametrization strategy. (6.6) was solved by using an explicit RK4 scheme with $\Delta t=5 \times 10^{-4}$ (continuous line), while for the reduced system the time step was chosen either as $\Delta t=1.5 \times 10^{-2}$ (crosses) or $\Delta t=2 \times 10^{-2}$ (circles). The initial condition is: $c_{0}=[0.0440,0.0177,0.861,0.188,0.102,0.00146]$.

\subsubsection{D grid based integrator: local parametrization}

The local parametrization approach represents an option whenever the mapping (7.10) is not unique. In this case, the parametrization matrix $R$ can be chosen such that its rows almost span the slow subspace around the initial condition $c_{0}$

$$
\boldsymbol{R}=\chi\left[\begin{array}{l}
\boldsymbol{c}(i+1, j+1)-c(i, j) \\
\boldsymbol{c}(i, j+1)-\boldsymbol{c}(i+1, j)
\end{array}\right]
$$

where $\chi$ is an arbitrary parameter and $\left(\xi_{0}, \eta_{0}\right)^{T}=\boldsymbol{R} \boldsymbol{c}_{0}^{T}$. By keeping the matrix (7.15) constant during the evaluation of the next pair $\left(\xi_{1}, \eta_{1}\right)$ after a time step $\Delta t$, the procedure described above in the present section is applied. At that point, only if $\left(\xi_{1}, \eta_{1}\right)$ has left the initial cell, the matrix $R$ is updated according to $(7.15)$, where $(i, j)$ denotes the down-left 
node of the new cell which $\left(\xi_{1}, \eta_{1}\right)$ belongs to. Similarly, numerical integration is carried on from $\left(\xi_{1}, \eta_{1}\right)$ to $\left(\xi_{2}, \eta_{2}\right)$ and so forth. The system (7.11) was solved, by using the local approach and by choosing bilinear interpolation on the four-nodes cell of Fig. 9 in order to reconstruct the 2-dimensional slow invariant manifold of (6.6) from the corresponding invariant grid. Computations were performed under the conditions of Fig. 10, and the comparison shows similar results compared to the global parameterization.

Remark 7.1. Notice that, a set of independent rows for the matrix (7.15), approximating the tangent space to a 2D manifold around $c_{0}$, can be also found as follows:

$$
\boldsymbol{R}=\chi\left[\begin{array}{l}
\boldsymbol{c}(i+1, j)-\boldsymbol{c}(i, j) \\
\boldsymbol{c}(i, j+1)-\boldsymbol{c}(i, j)
\end{array}\right]
$$

The matrix $R$ in the form (7.16) proves particularly convenient for parameterization of $q$-dimensional manifolds. In the latter case, the generalized $q \times n$ matrix $R$ can be written as follows:

$$
\boldsymbol{R}=\chi\left[\begin{array}{c}
\boldsymbol{c}(i+1, j, \cdots, k)-\boldsymbol{c}(i, j, \cdots, k) \\
\boldsymbol{c}(i, j+1, \cdots, k)-\boldsymbol{c}(i, j, \cdots, k) \\
\cdots \cdots \\
\boldsymbol{c}(i, j, \cdots, k+1)-\boldsymbol{c}(i, j, \cdots, k)
\end{array}\right]
$$

\section{The initial condition problem}

In general, when the initial condition is not on the slow invariant manifold, there is a need to find its image (on the SIM) after a fast transient before integrating the reduced set of equations. Toward this end, first we consider the fast foliation problem (Section 8.1), where we assume that the invariant manifold of slow motions is constructed, and the fibers of fast motions in its neighborhood are defined with the help of the thermodynamic projector. Second, the projection problem (Section 8.2) can be addressed as inverse to the fast foliation problem.

Let the initial condition of (6.2) $c_{0}$ not belong to the slow invariant manifold. In this case, the reduced initial condition $\xi_{0}$ can be expressed by the relation

$$
\xi_{0}=R c^{*},
$$

where $c^{*}$ is an appropriate point on the SIM in the region where the solution trajectory approaches it. If the dynamical system is supported by a Lyapunov function $G$, then each solution trajectory starts from $c_{0}$ and reaches the manifold in a neighborhood of a point where the fast fibers are defined by the null space $(\operatorname{ker} P)$ of the thermodynamic projector (see Section 6.1). Based on the above observation, in the following, we impose that the vector $\left(\boldsymbol{c}_{0}-\boldsymbol{c}^{*}\right)$ belongs to $\operatorname{ker} \boldsymbol{P}$ evaluated at $\boldsymbol{c}^{*}$

$$
\boldsymbol{c}_{0}=c^{*}+\operatorname{ker} \boldsymbol{P}\left(c^{*}\right) .
$$



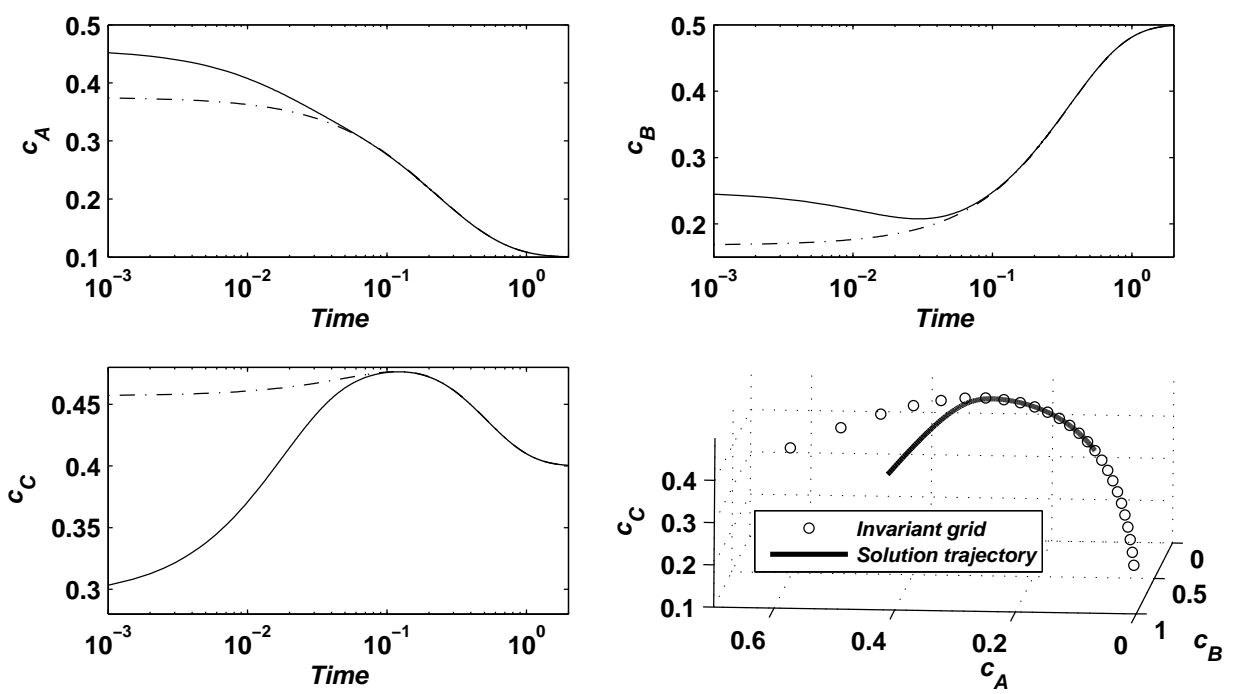

Figure 11: Solutions of (6.2) starting either from the invariant grid node $c^{*}=[0.375,0.168,0.457]$ (dashed line) or from the point $c_{0}$, evaluated through (8.3) with $\alpha^{\prime}=-0.2$ (continuous line).

\subsection{The fast foliation problem}

Let $c^{*}$ be an invariant grid node. By using the projector (6.9a), for Example 1, the condition (8.2) takes the form

$$
c_{0}=c^{*}+\alpha^{\prime} f_{1}
$$

where $\alpha^{\prime}$ is an arbitrary coefficient and $f_{1}$ the unit vector spanning the null space of the matrix

$$
\left[\begin{array}{ccc}
\ln \left(c_{A}^{*} / c_{A}^{e q}\right) & \ln \left(c_{B}^{*} / c_{B}^{e q}\right) & \ln \left(c_{C}^{*} / c_{C}^{e q}\right) \\
1 & 1 & 1
\end{array}\right]
$$

where the second row of (8.4) is due to the atom conservation. In Fig. 11 the comparison between the solutions of the full system (6.2), starting from both $c_{0}$ and the point $c^{*}$, is reported. The results show that, after a short arrival time $t^{*}$, the two solutions join each other. In the case of Example 2, the affine fast subspace (8.2) can be written as:

$$
c_{0}=c^{*}+\alpha^{\prime} f_{1}+\alpha^{\prime \prime} f_{2} .
$$

Here, the point $c^{*}$ belongs to the invariant grid of Fig. 4 , while the two unit vectors $f_{1}$ and $f_{2}$ span the null space of

$$
\left[\begin{array}{cccccc}
\ln \left(\frac{c_{H_{2}}^{*}}{c_{H_{2}}^{c}}\right) & \ln \left(\frac{c_{\mathrm{O}_{2}}^{*}}{c_{\mathrm{O}_{2}}^{c q}}\right) & \ln \left(\frac{c_{\mathrm{H}_{2} \mathrm{O}}^{*}}{c_{H_{2} \mathrm{O}}}\right) & \ln \left(\frac{c_{H}^{*}}{c_{H}^{c q}}\right) & \ln \left(\frac{c_{\mathrm{O}}^{*}}{c_{\mathrm{O}}^{c q}}\right) & \ln \left(\frac{c_{\mathrm{OH}}^{*}}{c_{\mathrm{OH}}^{c q}}\right) \\
\left(\frac{k_{1}}{c_{\mathrm{H}_{2}}^{*}}\right) & \left(\frac{k_{2}}{c_{\mathrm{O}_{2}}^{*}}\right) & \left(\frac{k_{3}}{c_{H_{2} \mathrm{O}}^{*}}\right) & \left(\frac{k_{4}}{c_{H}^{*}}\right) & \left(\frac{k_{5}}{c_{\mathrm{O}}^{*}}\right) & \left(\frac{k_{6}}{c_{\mathrm{OH}}^{*}}\right) \\
2 & 0 & 2 & 1 & 0 & 1 \\
0 & 2 & 1 & 0 & 1 & 1
\end{array}\right],
$$



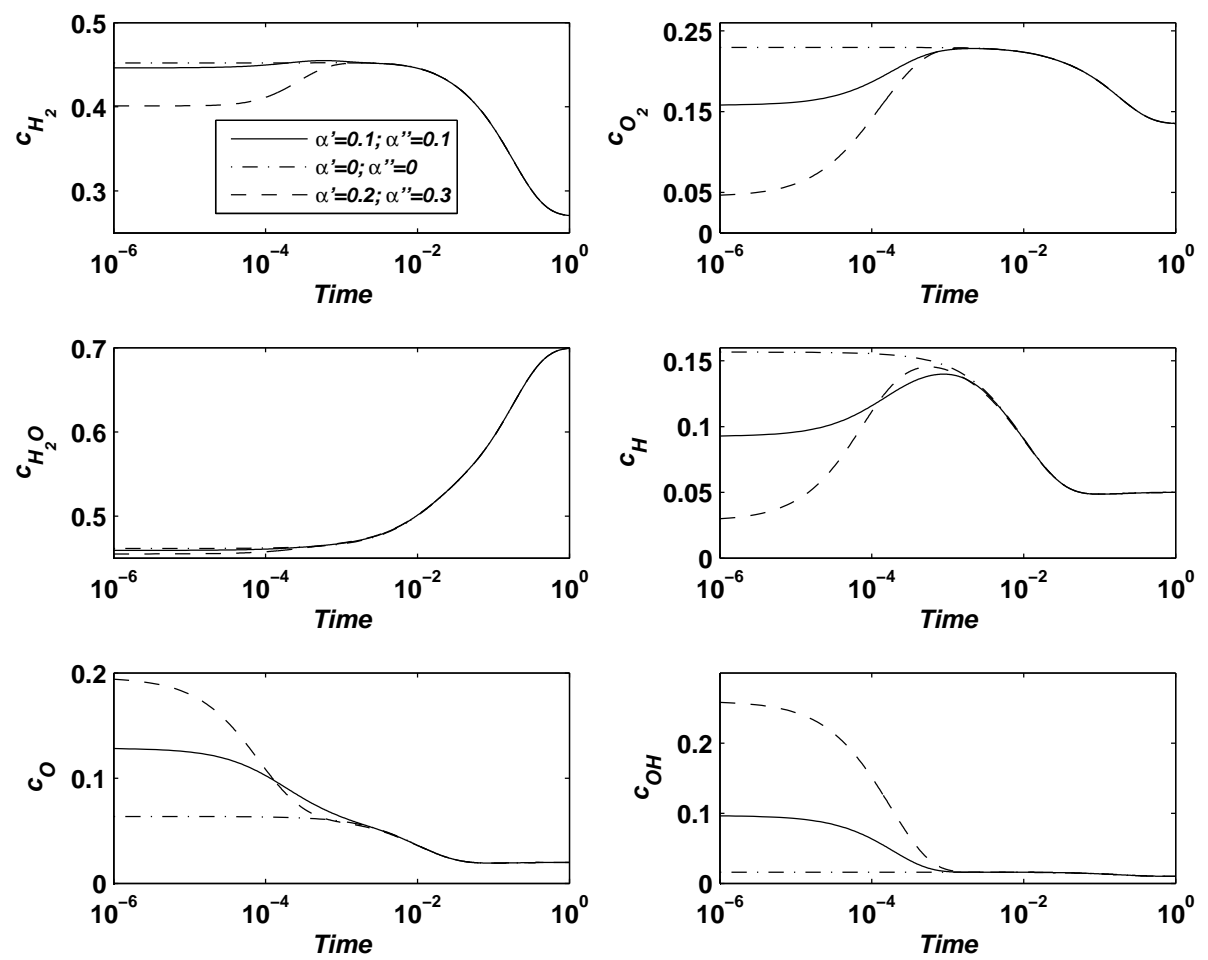

Figure 12: Solutions of (6.6) starting from the initial conditions $c_{0}$ corresponding to three different pairs $\left(\alpha^{\prime}, \alpha^{\prime \prime}\right)$ in (8.5) with $c^{*}=[0.452,0.229,0.462,0.157,0.0636,0.0160]$.

where the vector $k=\left[k_{1}, k_{2}, k_{3}, k_{4}, k_{5}, k_{6}\right]$ is evaluated according to (6.10). As shown in Fig. 12, a solution starting from $c_{0}$ meets the one that moves along the invariant grid $\left(\alpha^{\prime}=0, \alpha^{\prime \prime}=0\right)$, after an initial fast dynamics.

\subsection{The projection problem}

In general, because of the non-trivial dependence of the thermodynamic projector $\boldsymbol{P}$ on the state $c$, the node $c^{*}\left(c_{0}\right)$ cannot be found by a direct solving of (8.2). Therefore, here we employed an iterative procedure, which will be illustrated below with the help of Example 2. Iterations start after fixing two arbitrary directions: $f_{1}^{0}$ and $f_{2}^{0}$. The hypothetic fast subspace, passing through the initial condition $c_{0}$, is described by the equation (see (8.5))

$$
c=c_{0}-\alpha^{\prime} f_{1}^{0}-\alpha^{\prime \prime} f_{2}^{0}
$$

Let $c_{0}^{*}$ be the intersection point where the affine subspace (8.7) meets the invariant manifold, and let $\left\{f_{1}^{1}, f_{2}^{1}\right\}$ be a basis of the null space of the matrix (8.6) evaluated at $c_{0}^{*}$.

If the latter directions are a linear combination of the pair $\left\{f_{1}^{0}, f_{2}^{0}\right\}$, then the procedure is terminated and $c^{*}=c_{0}^{*}$. When this does not occur, a new intersection point $c_{1}^{*}$ between the fast subspace (8.7) and the manifold needs to be found. This time the right-hand side 
of (8.7) is updated with the new directions $\left\{f_{1}^{1}, f_{2}^{1}\right\}$, iterations are carried on accordingly and stopped as soon as the vectors $\left\{f_{1}^{i}, f_{2}^{i}\right\}$ result linearly dependent on the previous ones $\left\{f_{1}^{i-1}, f_{2}^{i-1}\right\}$ : In the latter case, we impose $c^{*}=c_{i}^{*}$.

For practical reasons, it proves convenient to set a small positive value $\varepsilon_{\lambda}$ as a threshold, and consider the following matrix

$$
A=\left[\begin{array}{llll}
f_{1}^{i-1} & f_{2}^{i-1} & f_{1}^{i} & f_{2}^{i}
\end{array}\right],
$$

whose columns are given by the hypothetic fast directions $f$ at the $i$-th step and at the previous one. For converging iterations, when two eigenvalues of the square matrix $A^{T} A$ (Gauss transformation of $A$ ) are smaller (by absolute value) than $\varepsilon_{\lambda}$, the procedure is terminated and $c_{i}^{*}$ is picked as approximate solution of (8.2). However, the method described above still applies if, at the $i$-th step, the basis $\left\{f_{1}^{i}, f_{2}^{i}\right\}$ is replaced by the fast eigenvectors of Jacobian matrix at the node $c_{i}^{*}$.

The described procedure was used to estimate $c^{*}$ corresponding to the initial condition $c_{0}=[0.285,0.04,0.6,0.08,0.17,0.15]$. Here, the two fastest eigenvectors of the Jacobian matrix, evaluated at the equilibrium point $c^{e q}$, were chosen as initial directions $\left\{f_{1}^{0}, f_{2}^{0}\right\}$, while the threshold was set up as $\varepsilon_{\lambda}=10^{-6}$. In this case, three iterations are needed till the method converges, and solutions of the system (6.6), starting from both $c_{0}$ and $c^{*}$, are reported in Fig. 13. As expected, they meet each other after an arrival time $t^{*}$ is elapsed.

In order to find the intersection point between the affine subspace (8.7) and the invariant manifold, the approximation (7.12) is used

$$
\boldsymbol{c}=w_{A} \boldsymbol{c}_{A}+w_{B} \boldsymbol{c}_{B}+w_{C} \boldsymbol{c}_{C},
$$

where $A, B, C$ are only three out of the four nodes of the grid cell of Fig. 9. In this case, the intersection point is solution of the linear system

$$
\left\{\begin{array}{l}
c_{0}(1)-\alpha^{\prime} f_{1}(1)-\alpha^{\prime \prime} f_{2}(1)=w_{A} c_{A}(1)+w_{B} c_{B}(1)+w_{C} c_{C}(1), \\
c_{0}(2)-\alpha^{\prime} f_{1}(2)-\alpha^{\prime \prime} f_{2}(2)=w_{A} c_{A}(2)+w_{B} c_{B}(2)+w_{C} c_{C}(2), \\
c_{0}(3)-\alpha^{\prime} f_{1}(3)-\alpha^{\prime \prime} f_{2}(3)=w_{A} c_{A}(3)+w_{B} c_{B}(3)+w_{C} c_{C}(3), \\
c_{0}(4)-\alpha^{\prime} f_{1}(4)-\alpha^{\prime \prime} f_{2}(4)=w_{A} c_{A}(4)+w_{B} c_{B}(4)+w_{C} c_{C}(4), \\
w_{A}+w_{B}+w_{C}=1,
\end{array}\right.
$$

where the unknowns are $0 \leq w_{A} \leq 1,0 \leq w_{B} \leq 1,0 \leq w_{C} \leq 1, \alpha^{\prime}$ and $\alpha^{\prime \prime}$. The first four equations of (8.10) are given by comparing (8.9) with (8.7). Note that here, because of the conservation equations (6.5), only four independent conditions can be imposed.

\section{Conclusions}

In this paper, following the MIG method we present in detail the entire process aiming at reducing descriptions in chemical kinetics. In particular, we focus on efficient techniques for invariant grid parameterization when solving the reduced dynamical system. 

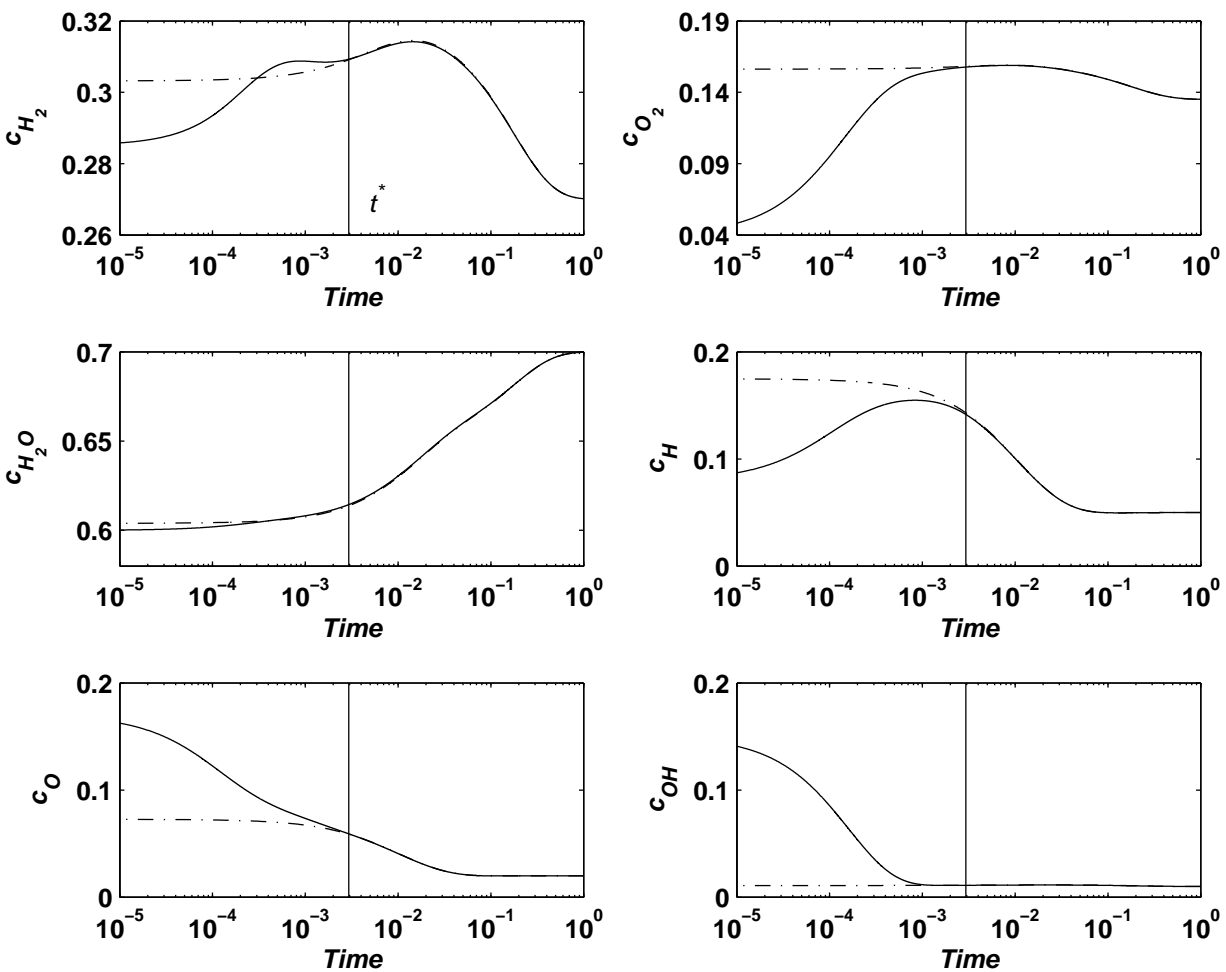

Figure 13: Solutions of the system (6.6) starting from the out-manifold point $c_{0}=[0.285,0.04,0.6,0.08,0.17,0.15]$ (continuous curve) and from the corresponding $c^{*}=[0.303,0.156,0.604,0.175,0.0726,0.0109]$ (dashed curve). The latter point was attained by iteratively solving the Eq. (8.5). The vertical line is drawn at the arrival time $t^{*}$.

The suggested procedures are validated in the case of a reaction mechanism for hydrogen oxidation, where comparisons between detailed and reduced models show excellent agreement. In particular, it has been demonstrated that thermodynamics enables to define the reduced system of equations and to cope with the stiffness of the detailed model. Moreover, based on the notion of thermodynamic projector, here we introduce the equation for computing reduced initial conditions and discuss a possible approach to solve it.

\section{Acknowledgments}

This work was partially supported by SNF (Project 200021-107885/1) (E.C.) and CCEMCH (I.V.K.).

\section{References}

[1] H. Rabitz, Chemical dynamics and kinetics phenomena as revealed by sensitivity analysis techniques, Chem. Rev., 87 (1987), 101-112. 
[2] A. S. Tomlin, T. Turanyi, M. J. Pilling, Mathematical tools for the construction, investigation and reduction of combustion mechanisms, In M. J. Pilling editor, Low-Temperature Combustion and Autoignition, Elsevier, 293-437.

[3] V. I. Bykov, G. S. Yablonskii, T. A. Akramov, The rate of the free energy decrease in the course of the complex chemical reaction, Dokl. Akad. Nauk SSSR (Doklady Chemistry), 234 (3) (1977), 621-634.

[4] V. I. Dimitrov, Partial kinetic models in the $\mathrm{H}_{2}-\mathrm{O}_{2}$ system, React. Kinet. Catal. Lett., 7 (1) (1977), 111-114.

[5] C. E. Frouzakis, K. Boulouchos, Analysis and reduction of the $\mathrm{CH}_{4}$ mechanism at lean condition, Comb. Sci. Tech., 159 (2000), 281-303.

[6] A. N. Gorban, I. V. Karlin, Method of invariant manifold for chemical kinetics, Chem. Eng. Sci., 58 (2003), 4751-4768.

[7] A. N. Gorban, I. V. Karlin, A. Y. Zinovyev, Invariant grids for reaction kinetics, Phys. A., 333 (2004), 106-154.

[8] F. A. Williams, Combustion Theory, The Fundamental Theory of Chemically Reactive Flow Systems, 2nd Edition, Addison-Wesley Publishing Company (1985).

[9] N. Kazantzis, Singular PDEs and the problem of finding invariant manifolds for nonlinear dynamical systems, Phys. Lett. A, 272 (2000), 257-263.

[10] P. Constantin, C. Foias, B. Nicolaenko, R. Temam, Integral Manifolds and Inertial Manifolds for Dissipative Partial Differential Equations, Appl. Math. Sci., 70 (1988). (Springer Verlag, New York).

[11] J. C. Robinson, A concise proof of the "geometric" construction of inertial manifolds, Phys. Lett. A., 200 (1995), 415-417.

[12] A. Debussche, R. Temam, Inertial manifolds and slow manifolds, Appl. Math. Lett., 4 (1991), 73-76.

[13] L. B. Ryashko, E. E. Shnol, On exponentially attracting invariant manifolds of ODEs, Nonlinearity., 16 (2003), 147-160.

[14] R. S. MacKay, Slow Manifolds, in: Energy Localisation and Transfer, ed. by T. Dauxois, A. Litvak-Hinenzon, R. S. MacKay, A. Spanoudaki, 149-192, (World. Sci., 2004).

[15] A. G. Ramm, Invariant manifolds for dissipative systems, J. Math. Phys., 50 (2009), 042701.

[16] E. Chiavazzo, A. N. Gorban, I. V. Karlin, Comparison of invariant manifolds for model reduction in chemical kinetics, Comm. Comput. Phys., 2 (2007), 964-992.

[17] A. N. Gorban, I. V. Karlin, Invariant Manifolds for Physical and Chemical Kinetics (Lecture Notes in Physics), Springer Berlin Heidelberg, 2005.

[18] E. Chiavazzo, I. V. Karlin, Quasi-equilibrium grid algorithm: geometric construction for model reduction, J. Comput. Phys., 227 (2008), 5535-5560.

[19] E. Chiavazzo, I. V. Karlin, C. E. Frouzakis, K. Boulouchos, Method of invariant grid for model reduction of hydrogen combustion, Proc. Combust. Instit., 32 (2009), 519-526.

[20] E. Chiavazzo, I. V. Karlin, A. N. Gorban, K. Boulouchos, Combustion simulation via lattice Boltzmann and reduced chemical kinetics, J. Stat. Mech.: Theory Exp., P06013 (2009).

[21] E. Chiavazzo, Invariant Manifolds and Lattice Boltzmann Method for Combustion, PhD Thesis 18233, Swiss Federal Institute of Technology, ETH-Zurich (2009).

[22] A. N. Gorban, I. V. Karlin, V. B. Zmievskii, T. F. Nonnenmacher, Relaxational trajectories: global approximations, Phys. A., 231 (1996), 648-672.

[23] A. N. Gorban, I.V.Karlin, Thermodynamic parametrization, Phys. A., 190 (1992), 393-404.

[24] A.N. Gorban, I.V. Karlin, Method of invariant manifolds and regularization of acoustic spectra, Transport. Theor. Stat., 23 (1994), 559-632. 
[25] A. N. Gorban, I. V. Karlin, Uniqueness of thermodynamic projector and kinetic basis of molecular individualism, Phys. A., 336 (2004), 391-432.

[26] R. J. Kee, G. Dixon-Lewis, J. Warnatz, M. E. Coltrin, J. A. Miller, A Fortran computer code package for the evaluation of gas-phase multicomponent transport properties, Sandia National Laboratories Technical Report, SAND86-8246, Albuquerque, NM, (1986).

[27] U. Maas, S. B. Pope, Simplifying chemical kinetics: intrinsic low-dimensional manifolds in composition space, Combust. Flame., 88 (1992), 239-264.

[28] V. Bykov, I. Goldfarb, V. Gol'dshtein, U. Maas, On a modified version of ILDM approach: asymptotic analysis based on integral manifolds, IMA J. Appl. Math., 71 (2006), 359-382.

[29] S. H. Lam, D. A. Goussis, Conventional asymptotic and computational singular perturbation for symplified kinetics modelling, in: M.O. Smooke (Ed.), Reduced Kinetic Mechanisms and Asymptotic Approximations for Methane-Air Flames, Springer Lecture Notes, (Springer Berlin 1991), pp. 227-242.

[30] S. H. Lam, D. A. Goussis, The CSP method for simplifying kinetics, Int. J. Chem. Kinet., 26 (1994), 461-486.

[31] D. A. Goussis, M. Valorani, An efficient iterative algorithm for the approximation of the fast and slow dynamics of stiff systems, J. Comput. Phys., 214 (2006), 316-346.

[32] Z. Ren, S. B. Pope, A. Vladimirsky, J. M. Guckenheimer, The invariant constrained equilibrium edge preimage curve method for the dimension reduction of chemical kinetics, $\mathrm{J}$. Chem. Phys., 124 (2006), 114111.

[33] I. G. Kevrekidis, C. W. Gear, J. M. Hyman, P. G. Kevrekidis, O. Runborg, C. Theodoropoulos, Equation-free: coarse-grained multiscale computation enabling microscopic simulators to perform system-level analysis, Comm. Math. Sci., 1 (2003), 715-762.

[34] C. W. Gear, T. J. Kaper, I. G. Kevrekidis, A. Zagaris, Projecting to a slow manifold: singularly perturbed systems and legacy codes, SIAM J. Appl. Dyn. Syst., 4 (2005), 711-732.

[35] D. Lebiedz, Computing minimal entropy production trajectories: an approach to model reduction in chemical kinetics, J. Chem. Phys., 120 (2004), 6890-6897.

[36] V. Reinhardt, M. Winckler, D. Lebiedz, Approximation of slow attracting manifolds in chemical kinetics by trajectory-based optimization approaches, J. Phys. Chem. A., 112 (2008), 1712-1718.

[37] K. D. Mease, S. Bharadwaj, S. Iravanchy, Timescale analysis for nonlinear dynamical systems, J. Guid. Control. Dynam., 26 (2003), 318-330.

[38] O. C. Zienkiewicz, R. L. Taylor, The Finite Element Method, Sixth Edition, ButterworthHeinemann (2005).

[39] A. M. Lyapunov, The General Problem of the Stability of Motion, Taylor \& Francis, London, (1992).

[40] R. T. Rockafellar, Convex Analysis, Princeton Landmarks in Mathematics, (Princeton University Press, New Jersey U.S.A.) (1996).

[41] J. C. Keck and D. Gillespie, Rate-controlled partial-equilibrium method for treating reacting gas-mixtures, Combust. Flame., 17 (1971), 237-241.

[42] D. Hamiroune, P. Bishnu, M. Metghalchi, J. C. Keck, Rate-controlled constrainedequilibrium method using constraint potentials, Combust. Theor. Model., 2 (1998), 81-94.

[43] Q. Tang, S. B. Pope, A more accurate projection in the rate-controlled constrainedequilibrium method for dimension reduction of combustion chemistry, Combust. Theor. Model., 8 (2004), 255-279. 